\title{
Synthesis and antimicrobial evaluation of some 6-aryl-5-cyano-2-thiouracil derivatives
}

\author{
MOSAAD SAYED MOHAMED* \\ SAMIR MOHAMED AWAD \\ NAGLAA MOHAMED AHMED \\ Organic Chemistry Department \\ Faculty of Pharmacy, Helwan University \\ Cairo, Egypt
}

Accepted May 9, 2011

\begin{abstract}
A series of 6-aryl-5-cyano-2-thiouracil derivatives (1a-d) was synthesized by the reaction of ethyl cyanoacetate with thiourea and aldehydes. These products were used as intermediate compounds for the synthesis of a number of thiouracil derivatives (2a-d to 10a-d). All compounds were screened for antibacterial and antifungal activities. Some of the prepared compounds, 6-(4-fluorophenyl)-4-oxo-2-thioxo-1,2,3,4-tetrahydropyrimidine-5-carboxamide (2a), 4-oxo-2-thioxo-6-(3,4,5-trimethoxyphenyl)-1,2,3,4-tetrahydropyrimidine-5-carboxamide (2d), 6-(4-fluorophenyl)-4-hydrazino-2-thioxo-1,2-dihydropyrimidine-5-cabonitrile (7a) and 4-hydrazino-2-thioxo-6-(3,4,5-trimethoxyphenyl)-1,2-dihydropyrimidine-5-carbonitrile (7d) revealed promising antimicrobial activity.
\end{abstract}

Keywords: 6-aryl-5-cyano-2-thiouracil, antibacterial activity, antifungal activity

It is well known that pyrimidine derivatives are of great biological interest, especially as antimicrobial, antiviral, anti-inflammatory and antitumor agents (1-7). Some series of pyrimido[4,5- $d$ ] pyrimidine-2,5-dione derivatives show antimicrobial activity against Staphylococcus aureus, Bacillus subtilis, Escherichia coli and antifungal activity against Candida albicans and Aspergillus niger, as proved by Sharma et al. (8). Bacimethrin (5-hydroxymethyl-2-methoxy-pyrimidin-4-amine) is a pyrimidine antibiotic active against several staphylococcal infections (9). 2-Thiouracils and 6-aryl-2-thiouracils are well known for their antifungal, antibacterial, anticancer and antiviral activity (10-12). The aim of this study was to synthesize new 6-aryl-5-cyano-2-thiouracil derivatives and to investigate their antimicrobial activity.

\footnotetext{
* Correspondence; e-mail: doctor-mosaad@hotmail.com
} 


\section{EXPERIMENTAL}

All melting points were uncorrected and were measured using an Electro-thermal IA9100 apparatus (Shimadzu, Japan). Microanalytical data were performed using a Vario, Elementar apparatus (Shimadzu). The IR spectra $(\mathrm{KBr})$ were recorded on a Perkin-Elmer1650 spectrophotometer (USA). ${ }^{1} \mathrm{H}$ NMR and ${ }^{13} \mathrm{C}$ NMR spectra were recorded on a Varian Mercury (300 MHz) spectrometer (Varian, UK) and the chemical shifts were expressed in ppm relative to TMS as internal reference. Mass spectra were recorded on a 70 eV EI Ms-QP 1000 EX (Shimadzu).

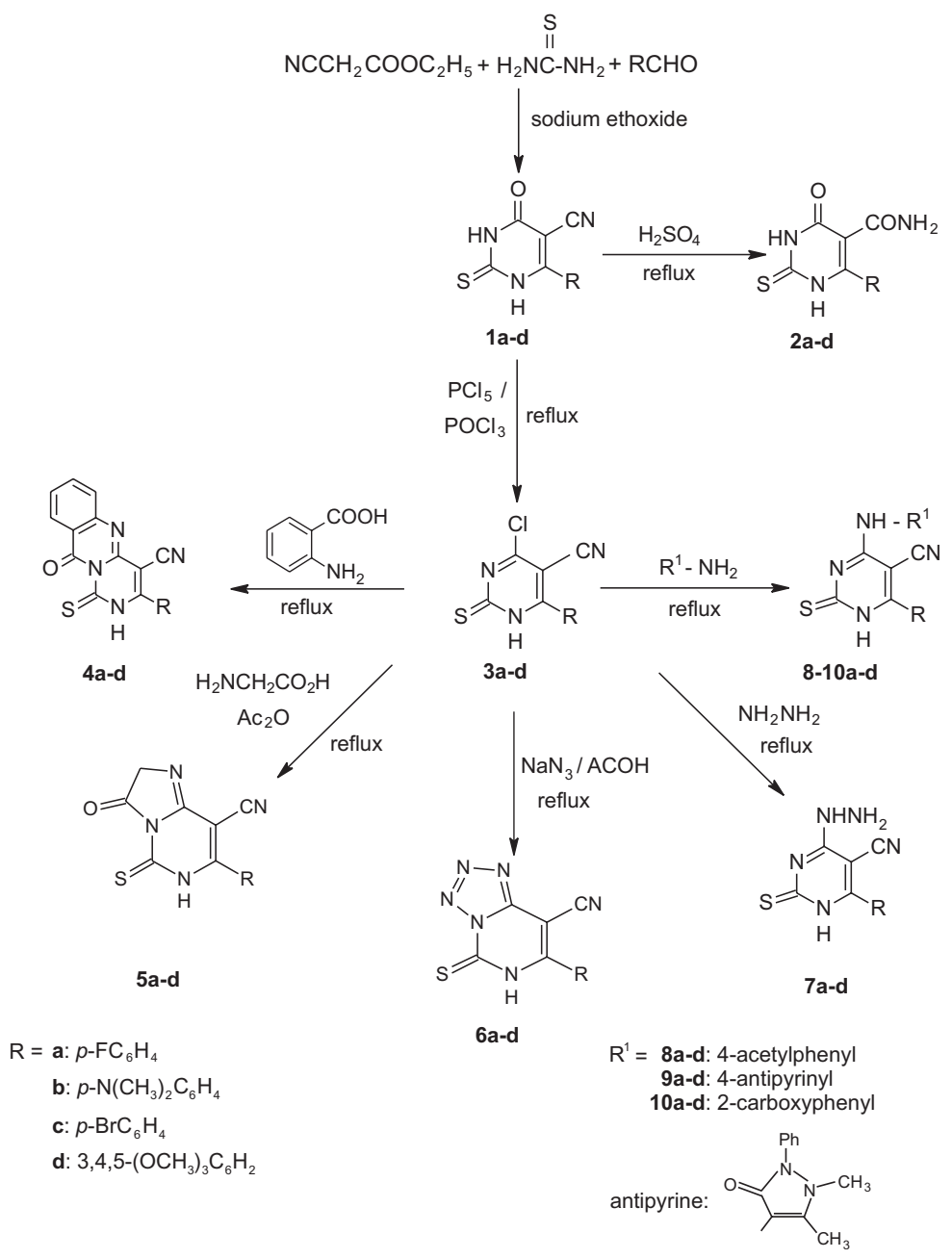

Shema 1 
M. S. Mohamed et al.: Synthesis and antimicrobial evaluation of some 6-aryl-5-cyano-2-thiouracil derivatives, Acta Pharm. 61 (2011) $171-185$.

Table I. Elemental analysis of the newly prepared compounds 1-10

\begin{tabular}{|c|c|c|c|c|c|c|}
\hline \multirow{2}{*}{ Compd. } & \multirow{2}{*}{$\begin{array}{l}\text { Yield } \\
(\%)\end{array}$} & \multirow{2}{*}{$\begin{array}{l}\text { M.p. } \\
\left({ }^{\circ} \mathrm{C}\right)\end{array}$} & \multirow{2}{*}{$\begin{array}{l}\text { Mol. formula } \\
\qquad\left(M_{\mathrm{r})}\right.\end{array}$} & \multicolumn{3}{|c|}{ Elemental analysis calcd./found(\%) } \\
\hline & & & & $\mathrm{C}$ & $\mathrm{H}$ & $\mathrm{N}$ \\
\hline $1 a$ & 80 & $235-236$ & $\begin{array}{c}\mathrm{C}_{11} \mathrm{H}_{6} \mathrm{FN}_{3} \mathrm{OS} \\
(247.24)\end{array}$ & $\begin{array}{l}53.44 \\
53.58\end{array}$ & $\begin{array}{l}2.45 \\
2.38\end{array}$ & $\begin{array}{l}17.00 \\
17.09\end{array}$ \\
\hline $1 b$ & 74 & $230-232$ & $\begin{array}{c}\mathrm{C}_{13} \mathrm{H}_{12} \mathrm{~N}_{4} \mathrm{OS} \\
(272.32)\end{array}$ & $\begin{array}{l}57.34 \\
57.29\end{array}$ & $\begin{array}{l}4.44 \\
4.30\end{array}$ & $\begin{array}{l}20.57 \\
20.46\end{array}$ \\
\hline 1c & 83 & $225-226$ & $\begin{array}{c}\mathrm{C}_{11} \mathrm{H}_{6} \mathrm{BrN}_{3} \mathrm{OS} \\
(308.12)\end{array}$ & $\begin{array}{l}42.87 \\
42.95\end{array}$ & $\begin{array}{l}1.96 \\
2.09\end{array}$ & $\begin{array}{l}13.64 \\
13.76\end{array}$ \\
\hline 1d & 77 & 245-247 & $\begin{array}{c}\mathrm{C}_{14} \mathrm{H}_{13} \mathrm{~N}_{3} \mathrm{O}_{4} \mathrm{~S} \\
(319.33)\end{array}$ & $\begin{array}{l}52.66 \\
52.72\end{array}$ & $\begin{array}{l}4.10 \\
4.19\end{array}$ & $\begin{array}{l}13.16 \\
12.95\end{array}$ \\
\hline $2 a$ & 45 & 100-102 & $\begin{array}{c}\mathrm{C}_{11} \mathrm{H}_{8} \mathrm{FN}_{3} \mathrm{O}_{2} \mathrm{~S} \\
(265.26)\end{array}$ & $\begin{array}{l}49.81 \\
49.70\end{array}$ & $\begin{array}{l}3.04 \\
2.90\end{array}$ & $\begin{array}{l}15.84 \\
15.72\end{array}$ \\
\hline $2 b$ & 48 & $118-120$ & $\begin{array}{c}\mathrm{C}_{13} \mathrm{H}_{14} \mathrm{~N}_{4} \mathrm{O}_{2} \mathrm{~S} \\
(290.34)\end{array}$ & $\begin{array}{l}53.78 \\
53.69\end{array}$ & $\begin{array}{l}4.86 \\
4.75\end{array}$ & $\begin{array}{l}19.30 \\
19.41\end{array}$ \\
\hline $2 c$ & 45 & $158-160$ & $\begin{array}{c}\mathrm{C}_{11} \mathrm{H}_{8} \mathrm{BrN}_{3} \mathrm{O}_{2} \mathrm{~S} \\
(326.12)\end{array}$ & $\begin{array}{l}40.51 \\
40.66\end{array}$ & $\begin{array}{l}2.47 \\
2.56\end{array}$ & $\begin{array}{l}12.88 \\
12.73\end{array}$ \\
\hline $2 d$ & 51 & 180-182 & $\begin{array}{c}\mathrm{C}_{14} \mathrm{H}_{15} \mathrm{~N}_{3} \mathrm{O}_{5} \mathrm{~S} \\
(337.35)\end{array}$ & $\begin{array}{l}49.84 \\
49.72\end{array}$ & $\begin{array}{l}4.48 \\
4.57\end{array}$ & $\begin{array}{l}12.46 \\
12.67\end{array}$ \\
\hline $3 a$ & 92 & $158-160$ & $\begin{array}{c}\mathrm{C}_{11} \mathrm{H}_{5} \mathrm{ClFN}_{3} \mathrm{~S} \\
(265.69)\end{array}$ & $\begin{array}{l}49.73 \\
49.60\end{array}$ & $\begin{array}{l}1.90 \\
2.12\end{array}$ & $\begin{array}{l}15.82 \\
15.70\end{array}$ \\
\hline $3 b$ & 85 & 179-180 & $\begin{array}{c}\mathrm{C}_{13} \mathrm{H}_{11} \mathrm{ClN}_{4} \mathrm{~S} \\
(290.77)\end{array}$ & $\begin{array}{l}53.70 \\
53.81\end{array}$ & $\begin{array}{l}3.81 \\
3.75\end{array}$ & $\begin{array}{l}19.27 \\
19.35\end{array}$ \\
\hline $3 c$ & 83 & $118-120$ & $\begin{array}{c}\mathrm{C}_{11} \mathrm{H}_{5} \mathrm{BrClN}_{3} \mathrm{~S} \\
(326.59)\end{array}$ & $\begin{array}{l}40.45 \\
40.54\end{array}$ & $\begin{array}{l}1.54 \\
1.63\end{array}$ & $\begin{array}{l}12.87 \\
12.76\end{array}$ \\
\hline $3 d$ & 93 & $138-140$ & $\begin{array}{c}\mathrm{C}_{14} \mathrm{H}_{12} \mathrm{ClN}_{3} \mathrm{O}_{3} \mathrm{~S} \\
(337.78)\end{array}$ & $\begin{array}{l}49.78 \\
49.85\end{array}$ & $\begin{array}{l}3.58 \\
3.64\end{array}$ & $\begin{array}{l}12.44 \\
12.52\end{array}$ \\
\hline $4 a$ & 82 & 160-162 & $\begin{array}{c}\mathrm{C}_{18} \mathrm{H}_{9} \mathrm{FN}_{4} \mathrm{OS} \\
(348.35)\end{array}$ & $\begin{array}{l}62.06 \\
62.18\end{array}$ & $\begin{array}{l}2.60 \\
2.72\end{array}$ & $\begin{array}{l}16.08 \\
16.17\end{array}$ \\
\hline $4 b$ & 80 & 200-202 & $\begin{array}{c}\mathrm{C}_{20} \mathrm{H}_{15} \mathrm{~N}_{5} \mathrm{OS} \\
(373.43)\end{array}$ & $\begin{array}{l}64.33 \\
64.45\end{array}$ & $\begin{array}{l}4.05 \\
4.18\end{array}$ & $\begin{array}{l}18.75 \\
18.83\end{array}$ \\
\hline $4 c$ & 75 & 116-118 & $\begin{array}{c}\mathrm{C}_{18} \mathrm{H}_{9} \mathrm{BrN}_{4} \mathrm{OS} \\
(409.22)\end{array}$ & $\begin{array}{l}52.83 \\
52.75\end{array}$ & $\begin{array}{l}2.22 \\
2.35\end{array}$ & $\begin{array}{l}13.69 \\
13.78\end{array}$ \\
\hline $4 d$ & 75 & 218-20 & $\begin{array}{c}\mathrm{C}_{21} \mathrm{H}_{16} \mathrm{~N}_{4} \mathrm{O}_{4} \mathrm{~S} \\
(420.44)\end{array}$ & $\begin{array}{l}59.99 \\
60.10\end{array}$ & $\begin{array}{l}3.84 \\
3.72\end{array}$ & $\begin{array}{l}13.33 \\
13.42\end{array}$ \\
\hline $5 a$ & 70 & 190-192 & $\begin{array}{c}\mathrm{C}_{13} \mathrm{H}_{7} \mathrm{FN}_{4} \mathrm{OS} \\
(286.28)\end{array}$ & $\begin{array}{l}54.54 \\
54.66\end{array}$ & $\begin{array}{l}2.46 \\
2.58\end{array}$ & $\begin{array}{l}19.57 \\
19.66\end{array}$ \\
\hline $5 b$ & 73 & 195-197 & $\begin{array}{c}\mathrm{C}_{15} \mathrm{H}_{13} \mathrm{~N}_{5} \mathrm{OS} \\
(311.36)\end{array}$ & $\begin{array}{l}57.86 \\
57.98\end{array}$ & $\begin{array}{l}4.21 \\
4.37\end{array}$ & $\begin{array}{l}22.49 \\
22.35\end{array}$ \\
\hline $5 c$ & 70 & $200-202$ & $\begin{array}{c}\mathrm{C}_{13} \mathrm{H}_{7} \mathrm{BrN}_{4} \mathrm{OS} \\
(347.18)\end{array}$ & $\begin{array}{l}44.97 \\
44.78\end{array}$ & $\begin{array}{l}2.03 \\
1.86\end{array}$ & $\begin{array}{l}16.14 \\
16.28\end{array}$ \\
\hline $5 d$ & 72 & $208-210$ & $\begin{array}{c}\mathrm{C}_{16} \mathrm{H}_{14} \mathrm{~N}_{4} \mathrm{O}_{4} \mathrm{~S} \\
(358.37)\end{array}$ & $\begin{array}{l}53.62 \\
53.72\end{array}$ & $\begin{array}{l}3.94 \\
4.08\end{array}$ & $\begin{array}{l}15.63 \\
15.75\end{array}$ \\
\hline
\end{tabular}


M. S. Mohamed et al.: Synthesis and antimicrobial evaluation of some 6-aryl-5-cyano-2-thiouracil derivatives, Acta Pharm. 61 (2011) $171-185$.

\begin{tabular}{|c|c|c|c|c|c|c|}
\hline \multirow{2}{*}{ Compd. } & \multirow{2}{*}{$\begin{array}{l}\text { Yield } \\
(\%)\end{array}$} & \multirow{2}{*}{$\begin{array}{l}\text { M.p. } \\
\left({ }^{\circ} \mathrm{C}\right)\end{array}$} & \multirow{2}{*}{$\begin{array}{l}\text { Mol. formula } \\
\qquad\left(M_{\mathrm{r})}\right.\end{array}$} & \multicolumn{3}{|c|}{ Elemental analysis calcd./found(\%) } \\
\hline & & & & C & $\mathrm{H}$ & $\mathrm{N}$ \\
\hline $6 a$ & 72 & 200-202 & $\begin{array}{c}\mathrm{C}_{11} \mathrm{H}_{5} \mathrm{FN}_{6} \mathrm{~S} \\
(272.26)\end{array}$ & $\begin{array}{l}48.53 \\
48.66\end{array}$ & $\begin{array}{l}1.85 \\
1.96\end{array}$ & $\begin{array}{l}30.87 \\
30.75\end{array}$ \\
\hline $6 b$ & 70 & $210-212$ & $\begin{array}{c}\mathrm{C}_{13} \mathrm{H}_{11} \mathrm{~N}_{7} \mathrm{~S} \\
(297.33)\end{array}$ & $\begin{array}{l}52.51 \\
52.65\end{array}$ & $\begin{array}{l}3.73 \\
3.87\end{array}$ & $\begin{array}{l}32.97 \\
33.12\end{array}$ \\
\hline $6 c$ & 65 & 205-207 & $\begin{array}{c}\mathrm{C}_{11} \mathrm{H}_{5} \mathrm{BrN}_{6} \mathrm{~S} \\
(333.16)\end{array}$ & $\begin{array}{l}39.66 \\
39.78\end{array}$ & $\begin{array}{l}1.51 \\
1.66\end{array}$ & $\begin{array}{l}25.22 \\
25.10\end{array}$ \\
\hline $6 \mathrm{~d}$ & 75 & 180-182 & $\begin{array}{c}\mathrm{C}_{14} \mathrm{H}_{12} \mathrm{~N}_{6} \mathrm{O}_{3} \mathrm{~S} \\
(344.34)\end{array}$ & $\begin{array}{l}48.83 \\
48.96\end{array}$ & $\begin{array}{l}3.51 \\
3.42\end{array}$ & $\begin{array}{l}24.41 \\
24.53\end{array}$ \\
\hline $7 a$ & 65 & $212-214$ & $\begin{array}{c}\mathrm{C}_{11} \mathrm{H}_{8} \mathrm{FN}_{5} \mathrm{~S} \\
(261.27)\end{array}$ & $\begin{array}{l}50.57 \\
50.42\end{array}$ & $\begin{array}{l}3.09 \\
2.90\end{array}$ & $\begin{array}{l}26.80 \\
26.93\end{array}$ \\
\hline $7 \mathrm{~b}$ & 62 & $260-262$ & $\begin{array}{c}\mathrm{C}_{13} \mathrm{H}_{14} \mathrm{~N}_{6} \mathrm{~S} \\
(286.35)\end{array}$ & $\begin{array}{l}54.53 \\
54.70\end{array}$ & $\begin{array}{l}4.93 \\
5.15\end{array}$ & $\begin{array}{l}29.35 \\
29.24\end{array}$ \\
\hline $7 \mathrm{c}$ & 66 & 190-192 & $\begin{array}{c}\mathrm{C}_{11} \mathrm{H}_{8} \mathrm{BrN}_{5} \mathrm{~S} \\
(322.18)\end{array}$ & $\begin{array}{l}41.01 \\
41.18\end{array}$ & $\begin{array}{l}2.50 \\
2.64\end{array}$ & $\begin{array}{l}21.74 \\
21.62\end{array}$ \\
\hline $7 \mathrm{~d}$ & 68 & $215-217$ & $\begin{array}{c}\mathrm{C}_{14} \mathrm{H}_{15} \mathrm{~N}_{5} \mathrm{O}_{3} \mathrm{~S} \\
(333.36)\end{array}$ & $\begin{array}{l}50.44 \\
50.58\end{array}$ & $\begin{array}{l}4.54 \\
4.42\end{array}$ & $\begin{array}{l}21.01 \\
21.10\end{array}$ \\
\hline $8 a$ & 60 & $160-162$ & $\begin{array}{c}\mathrm{C}_{19} \mathrm{H}_{13} \mathrm{FN}_{4} \mathrm{OS} \\
(364.39)\end{array}$ & $\begin{array}{l}62.63 \\
62.75\end{array}$ & $\begin{array}{l}3.60 \\
3.52\end{array}$ & $\begin{array}{l}15.38 \\
15.26\end{array}$ \\
\hline $8 b$ & 62 & $188-190$ & $\begin{array}{c}\mathrm{C}_{21} \mathrm{H}_{19} \mathrm{~N}_{5} \mathrm{OS} \\
(389.47)\end{array}$ & $\begin{array}{l}64.76 \\
64.88\end{array}$ & $\begin{array}{l}4.92 \\
5.08\end{array}$ & $\begin{array}{l}17.98 \\
18.10\end{array}$ \\
\hline $8 c$ & 60 & $210-212$ & $\begin{array}{c}\mathrm{C}_{19} \mathrm{H}_{13} \mathrm{BrN}_{4} \mathrm{OS} \\
(425.30)\end{array}$ & $\begin{array}{l}53.66 \\
53.72\end{array}$ & $\begin{array}{l}3.08 \\
3.13\end{array}$ & $\begin{array}{l}13.17 \\
13.10\end{array}$ \\
\hline $8 d$ & 65 & 200-202 & $\begin{array}{c}\mathrm{C}_{22} \mathrm{H}_{20} \mathrm{~N}_{4} \mathrm{O}_{4} \mathrm{~S} \\
(436.48)\end{array}$ & $\begin{array}{l}60.54 \\
60.64\end{array}$ & $\begin{array}{l}4.62 \\
4.78\end{array}$ & $\begin{array}{l}12.84 \\
12.72\end{array}$ \\
\hline $9 a$ & 70 & 195-197 & $\begin{array}{c}\mathrm{C}_{22} \mathrm{H}_{17} \mathrm{FN}_{6} \mathrm{OS} \\
(432.47)\end{array}$ & $\begin{array}{l}61.10 \\
61.22\end{array}$ & $\begin{array}{l}3.96 \\
4.09\end{array}$ & $\begin{array}{l}19.43 \\
19.35\end{array}$ \\
\hline $9 b$ & 75 & $215-217$ & $\begin{array}{c}\mathrm{C}_{24} \mathrm{H}_{23} \mathrm{~N}_{7} \mathrm{OS} \\
(457.55)\end{array}$ & $\begin{array}{l}63.00 \\
63.09\end{array}$ & $\begin{array}{l}5.07 \\
5.18\end{array}$ & $\begin{array}{l}21.43 \\
21.32\end{array}$ \\
\hline $9 \mathrm{c}$ & 71 & $160-162$ & $\begin{array}{c}\mathrm{C}_{22} \mathrm{H}_{17} \mathrm{BrN}_{6} \mathrm{OS} \\
(493.37)\end{array}$ & $\begin{array}{l}53.56 \\
53.45\end{array}$ & $\begin{array}{l}3.47 \\
3.55\end{array}$ & $\begin{array}{l}17.03 \\
16.92\end{array}$ \\
\hline $9 d$ & 74 & $170-172$ & $\begin{array}{c}\mathrm{C}_{25} \mathrm{H}_{24} \mathrm{~N}_{6} \mathrm{O}_{4} \mathrm{~S} \\
(504.56)\end{array}$ & $\begin{array}{l}59.51 \\
59.40\end{array}$ & $\begin{array}{l}4.79 \\
4.67\end{array}$ & $\begin{array}{l}16.66 \\
16.78\end{array}$ \\
\hline $10 a$ & 70 & 180-182 & $\begin{array}{c}\mathrm{C}_{18} \mathrm{H}_{11} \mathrm{FN}_{4} \mathrm{O}_{2} \mathrm{~S} \\
(366.36)\end{array}$ & $\begin{array}{l}59.01 \\
59.12\end{array}$ & $\begin{array}{l}3.03 \\
3.11\end{array}$ & $\begin{array}{l}15.29 \\
15.15\end{array}$ \\
\hline $10 \mathrm{~b}$ & 75 & $165-167$ & $\begin{array}{c}\mathrm{C}_{20} \mathrm{H}_{17} \mathrm{~N}_{5} \mathrm{O}_{2} \mathrm{~S} \\
(391.44)\end{array}$ & $\begin{array}{l}61.37 \\
61.48\end{array}$ & $\begin{array}{l}4.38 \\
4.46\end{array}$ & $\begin{array}{l}17.89 \\
17.78\end{array}$ \\
\hline $10 \mathrm{c}$ & 72 & $170-172$ & $\begin{array}{c}\mathrm{C}_{18} \mathrm{H}_{11} \mathrm{BrN}_{4} \mathrm{O}_{2} \mathrm{~S} \\
(427.27)\end{array}$ & $\begin{array}{l}50.60 \\
50.72\end{array}$ & $\begin{array}{l}2.59 \\
2.67\end{array}$ & $\begin{array}{l}13.11 \\
13.04\end{array}$ \\
\hline $10 \mathrm{~d}$ & 77 & $160-162$ & $\begin{array}{c}\mathrm{C}_{21} \mathrm{H}_{18} \mathrm{~N}_{4} \mathrm{O}_{5} \mathrm{~S} \\
(438.45)\end{array}$ & $\begin{array}{l}57.53 \\
57.44\end{array}$ & $\begin{array}{l}4.14 \\
4.09\end{array}$ & $\begin{array}{l}12.78 \\
12.84\end{array}$ \\
\hline
\end{tabular}


Physicochemical and spectral data for the synthesized compounds are given in Tables I and II. Target compounds were synthesized as outlined in Scheme 1.

Table II. Spectral data of the newly prepared compounds 1-10

\begin{tabular}{|c|c|c|c|c|}
\hline Compd. & $\operatorname{IR}\left(\mathrm{cm}^{-1}\right)$ & $\left({ }^{1} \mathrm{H}\right.$ NMR) (ppm) & ${ }^{13} \mathrm{C}$ NMR (ppm) & $\begin{array}{c}\text { Mass }(m / z, \% \\
\text { abundance) }\end{array}$ \\
\hline $1 \mathrm{a}$ & $\begin{array}{l}3400-3000 \\
(2 \mathrm{NH}, \text { stretch- } \\
\text { ing) } 2230(\mathrm{CN}) \\
1690(\mathrm{C}=\mathrm{O})\end{array}$ & $\begin{array}{l}6.5-7.2(\mathrm{~m}, 4 \mathrm{H}, \mathrm{Ar}-\mathrm{H}), \\
10,10.7\left(\mathrm{~s}, 2 \mathrm{H}, 2 \mathrm{NH}, \mathrm{D}_{2} \mathrm{O}\right. \\
\text { exchangeable })\end{array}$ & $\begin{array}{l}175.1(\mathrm{C}=\mathrm{S}), 90.4,161.8 \\
\text { (thiouracil } \mathrm{C}=\mathrm{C}), 160 \\
(\mathrm{C}=\mathrm{O}), 114.9,125.3,131.2 \\
151.9 \text { (benzene } \mathrm{C}), 115.3 \\
(\mathrm{CN})\end{array}$ & $\begin{array}{l}247\left[\mathrm{M}^{+}\right] \\
(57.59 \%)\end{array}$ \\
\hline $1 b$ & $\begin{array}{l}3450-3050 \\
(2 \mathrm{NH}, \text { stretch- } \\
\text { ing) } 2200(\mathrm{CN}) \\
1680(\mathrm{C}=\mathrm{O})\end{array}$ & $\begin{array}{l}2.3,2.5\left(\mathrm{~s}, 6 \mathrm{H}, 2 \mathrm{CH}_{3}\right), \\
6.5-7.2(\mathrm{~m}, 4 \mathrm{H}, \mathrm{Ar}-\mathrm{H}), \\
10,10.5\left(\mathrm{~s}, 2 \mathrm{H}, 2 \mathrm{NH}, \mathrm{D}_{2} \mathrm{O}\right. \\
\text { exchangeable })\end{array}$ & $\begin{array}{l}177.2(\mathrm{C}=\mathrm{S}), 86.7,159.6 \\
\text { (thiouracil } \mathrm{C}=\mathrm{C}), 158.2 \\
(\mathrm{C}=\mathrm{O}), 113.8,127.9,130.4 \\
152.47 \text { (benzene } \mathrm{C}), 40.3 \\
\left(2 \mathrm{CH}_{3}\right), 115.2(\mathrm{CN})\end{array}$ & $\begin{array}{l}272\left[\mathrm{M}^{+}\right] \\
(100 \%)\end{array}$ \\
\hline 1c & $\begin{array}{l}3450-3015 \\
(2 \mathrm{NH}, \text { stretch- } \\
\text { ing) } 2235(\mathrm{CN}) \\
1685(\mathrm{C}=\mathrm{O})\end{array}$ & $\begin{array}{l}6.8-7.5(\mathrm{~m}, 4 \mathrm{H}, \mathrm{Ar}-\mathrm{H}) \\
10.2,11.1(\mathrm{~s}, 2 \mathrm{H}, 2 \mathrm{NH}, \\
\left.\mathrm{D}_{2} \mathrm{O} \text { exchangeable }\right)\end{array}$ & $\begin{array}{l}175.1(C=S), 92.2,160 \\
\text { (thiouracil } C=C), 158.5 \\
(C=O), 115,128.8,133.4 \\
150.3 \text { (benzene } C), 114.05 \\
(C N)\end{array}$ & $\begin{array}{l}307[\mathrm{M}+] \\
(19.6 \%) \\
309[\mathrm{M}+2] \\
(19.8 \%)\end{array}$ \\
\hline $1 d$ & $\begin{array}{l}3450-3110 \\
(2 \mathrm{NH}, \text { stretch- } \\
\text { ing) } 2215(\mathrm{CN}) \\
1675(\mathrm{C}=\mathrm{O})\end{array}$ & $\begin{array}{l}3.1-3.4\left(\mathrm{~s}, 9 \mathrm{H}, \mathrm{OCH}_{3}\right), \\
6.8(\mathrm{~s}, 2 \mathrm{H}, \mathrm{Ar}-\mathrm{H}), 10.2, \\
11.1\left(\mathrm{~s}, 2 \mathrm{H}, 2 \mathrm{NH}, \mathrm{D}_{2} \mathrm{O}\right. \\
\text { exchangeable })\end{array}$ & $\begin{array}{l}178.04(\mathrm{C}=\mathrm{S}), 88.7,159.7 \\
\text { (thiouracil C=C),157.9 } \\
(\mathrm{C}=\mathrm{O}), 114.8,130.4,151.7 \\
152(\text { benzene } \mathrm{C}), 55.4,55.7 \\
\left(3 \mathrm{CH}_{3}\right), 115.3(\mathrm{CN})\end{array}$ & $\begin{array}{l}319\left[\mathrm{M}^{+}\right] \\
(57.59 \%)\end{array}$ \\
\hline $2 a$ & $\begin{array}{l}3450-3010 \\
\left(\mathrm{NH}_{2}, 2 \mathrm{NH}\right) \\
\left.1660(\mathrm{CONH})_{2}\right) \\
1700(\mathrm{C}=\mathrm{O})\end{array}$ & $\begin{array}{l}5.1\left(\mathrm{~s}, 2 \mathrm{H}, \mathrm{CONH}_{2} \mathrm{D}_{2} \mathrm{O}\right. \\
\text { exchangeable), 7.2-8 } \\
(\mathrm{m}, 4 \mathrm{H}, \mathrm{Ar}-\mathrm{H}), 10.4, \\
11.5\left(\mathrm{~s}, 2 \mathrm{H}, 2 \mathrm{NH}, \mathrm{D}_{2} \mathrm{O}\right. \\
\text { exchangeable) }\end{array}$ & $\begin{array}{l}80.2,162.6 \text { (thiouracil } \\
C=C), 160(2 C=O), 175.9 \\
(C=S), 115.4,127.8,130.5 \\
161.3 \text { (benzene C) }\end{array}$ & $\begin{array}{l}265\left[\mathrm{M}^{+}\right] \\
(18 \%)\end{array}$ \\
\hline $2 b$ & $\begin{array}{l}3500-3020 \\
\left(\mathrm{NH}_{2}, 2 \mathrm{NH}\right) \\
1660\left(\mathrm{CONH}{ }_{2}\right) \\
1695(\mathrm{C}=\mathrm{O})\end{array}$ & $\begin{array}{l}2.3,2.5\left(\mathrm{~s}, 6 \mathrm{H}, 2 \mathrm{CH}_{3}\right) \\
5\left(\mathrm{~s}, 2 \mathrm{H}, \mathrm{CONH}_{2}, \mathrm{D}_{2} \mathrm{O} \text { ex- }\right. \\
\text { changeable }), 6.5-7.2(\mathrm{~m}, \\
4 \mathrm{H}, \mathrm{Ar}-\mathrm{H}, 10,11.5(\mathrm{~s}, 2 \mathrm{H}, \\
\left.\mathrm{N}-\mathrm{H}, \mathrm{D}_{2} \mathrm{O} \text { exchangeable }\right)\end{array}$ & $\begin{array}{l}82.4,161.8 \text { (thiouracil } \\
\mathrm{C}=\mathrm{C}), 160.9(2 \mathrm{C}=\mathrm{O}), 176.2 \\
(\mathrm{C}=\mathrm{S}), 113,124.4,127.1, \\
143.7 \text { (benzene } \mathrm{C}), 43.6 \\
\left(2 \mathrm{CH}_{3} \text { aliphatic) }\right.\end{array}$ & $\begin{array}{l}290\left[\mathrm{M}^{+}\right] \\
(23 \%)\end{array}$ \\
\hline $2 c$ & $\begin{array}{l}3455-3000 \\
\left(\mathrm{NH}_{2}, 2 \mathrm{NH}\right) \\
1664\left(\mathrm{CONH}{ }_{2}\right) \\
1704(\mathrm{C}=\mathrm{O})\end{array}$ & $\begin{array}{l}5.1\left(\mathrm{~s}, 2 \mathrm{H}, \mathrm{CONH}_{2}, \mathrm{D}_{2} \mathrm{O}\right. \\
\text { exchangeable }), 7.2-8(\mathrm{~m}, \\
4 \mathrm{H}, \mathrm{Ar}-\mathrm{H}), 10.4,11.5(\mathrm{~s}, 2 \mathrm{H}, \\
\left.2 \mathrm{NH}, \mathrm{D}_{2} \mathrm{O} \text { exchangeable }\right)\end{array}$ & $\begin{array}{l}85.1,163 \text { (thiouracil } C=C) \text {, } \\
160.9(2 \mathrm{C}=\mathrm{O}), 175.7(\mathrm{C}=\mathrm{S}) \\
122.3,128.4,131.7,133.9 \\
\text { (benzene } \mathrm{C} \text { ) }\end{array}$ & $\begin{array}{l}325\left[\mathrm{M}^{+}\right] \\
(15.3 \%) \\
327[\mathrm{M}+2] \\
(15.1 \%)\end{array}$ \\
\hline $2 d$ & $\begin{array}{l}3500-3100 \\
\left(\mathrm{NH}_{2}, 2 \mathrm{NH}\right) \\
\left.1660(\mathrm{CONH})_{2}\right) \\
1710(\mathrm{C}=\mathrm{O})\end{array}$ & $\begin{array}{l}3.8-4\left(\mathrm{~s}, 9 \mathrm{H}, 3 \mathrm{OCH}_{3},\right. \\
5.2\left(\mathrm{~s}, 2 \mathrm{H}, \mathrm{CONH}_{2}, \mathrm{D}_{2} \mathrm{O}\right. \\
\text { exchangeable }), 7.3(\mathrm{~s}, 2 \mathrm{H}, \\
\text { Ar- } \mathrm{H}), 11-11.6(\mathrm{~s}, 2 \mathrm{H}, \\
\left.2 \mathrm{NH}, \mathrm{D}_{2} \mathrm{O} \text { exchangeable }\right)\end{array}$ & $\begin{array}{l}80.9,161.8 \text { (thiouracil } \\
\mathrm{C}=\mathrm{C}), 160.9(2 \mathrm{C}=\mathrm{O}), 176.1 \\
(\mathrm{C}=\mathrm{S}), 115.1,129.2,132.4 \\
148.5 \text { (benzene } \mathrm{C}), 56.3 \\
\left(3 \mathrm{CH}_{3} \text { aliphatic) }\right.\end{array}$ & $\begin{array}{l}337\left[\mathrm{M}^{+}\right] \\
(17 \%)\end{array}$ \\
\hline
\end{tabular}


$3350(\mathrm{NH}$,

stretching)

$2230(\mathrm{CN})$

$1270(\mathrm{C}=\mathrm{S})$

3200 (NH,

$3 b$

stretching)

$2210(\mathrm{CN})$

$1275(\mathrm{C}=\mathrm{S})$

3300 (NH,

$3 c$

stretching)

$2215(\mathrm{CN})$

$1275(\mathrm{C}=\mathrm{S})$

3310 (NH,

$3 d$

stretching)

$2225(\mathrm{CN})$

$1272(\mathrm{C}=\mathrm{S})$

3000 (NH,

$4 a$

stretching)

$2200(\mathrm{CN})$

$1670(\mathrm{C}=\mathrm{O})$

3100 (NH,

$4 b$

stretching)

$2210(\mathrm{CN})$

$1675(\mathrm{C}=\mathrm{O})$

3120 (NH, stretching) $2215(\mathrm{CN})$ $1680(\mathrm{C}=\mathrm{O})$

3100 (NH,

4d

stretching)

$2225(\mathrm{CN})$

$1677(\mathrm{C}=\mathrm{O})$

$3300(\mathrm{NH}$,

stretching)

$2240(\mathrm{CN})$

$1670(\mathrm{C}=\mathrm{O})$
7.1-7.7 (m, 4H, Ar-H), $11.8\left(\mathrm{~s}, 1 \mathrm{H},-\mathrm{NH}, \mathrm{D}_{2} \mathrm{O}\right.$

exchangeable)

$2.8\left(6 \mathrm{H}, \mathrm{s}, 2 \mathrm{CH}_{3}\right), 7.1-7.4$

$(4 \mathrm{H}, \mathrm{m}, \mathrm{Ar}-\mathrm{H}), 11.2(1 \mathrm{H}, \mathrm{s}$,

$\mathrm{NH}, \mathrm{D}_{2} \mathrm{O}$ exchangeable)

$2.8\left(6 \mathrm{H}, \mathrm{s}, 2 \mathrm{CH}_{3}\right), 7.1-7.4$

$(4 \mathrm{H}, \mathrm{m}, \mathrm{Ar}-\mathrm{H}), 11.2(1 \mathrm{H}, \mathrm{s}$,

$\mathrm{NH}, \mathrm{D}_{2} \mathrm{O}$ exchangeable)

$7.4(\mathrm{~S}, 2 \mathrm{H}, \mathrm{Ar}-\mathrm{H})$, $10\left(\mathrm{~s}, 1 \mathrm{H},-\mathrm{NH}, \mathrm{D}_{2} \mathrm{O}\right.$ exchangeable)

6.7-7.6 (m, 8H, Ar-H), $11.2\left(\mathrm{~s}, 1 \mathrm{H},-\mathrm{NH}, \mathrm{D}_{2} \mathrm{O}\right.$ exchangeable)

2.5, $2.8\left(\mathrm{~s}, 6 \mathrm{H}, 2 \mathrm{CH}_{3}\right)$, 6.6-8 (m, 8H, Ar-H), $11.2\left(\mathrm{~s}, 1 \mathrm{H},-\mathrm{NH}, \mathrm{D}_{2} \mathrm{O}\right.$ exchangeable)

7-7.6 (m, 8H, Ar-H), $11.2\left(\mathrm{~s}, 1 \mathrm{H},-\mathrm{NH}, \mathrm{D}_{2} \mathrm{O}\right.$ exchangeable)

$3,3.5,3.8\left(\mathrm{~s}, 9 \mathrm{H}, 3 \mathrm{OCH}_{3}\right)$, 7.5-8.1 (m, 6H, Ar-H), 11.2 (s, 1H, - NH, $\mathrm{D}_{2} \mathrm{O}$ exchangeable)

6.8 (s, 2H, imidazo), 7.3-8 (m, 4H, Ar-H), $10.2\left(\mathrm{~s}, 1 \mathrm{H},-\mathrm{NH}, \mathrm{D}_{2} \mathrm{O}\right.$ exchangeable)
88.2, 160 (thiouracil $\mathrm{C}=\mathrm{C}$ ), $\quad 265\left[\mathrm{M}^{+}\right]$

$159.2(\mathrm{C}=\mathrm{N}), 180(\mathrm{C}=\mathrm{S}), \quad(100 \%)$, $115.4,127.8,130.5,161.3 \quad 267[\mathrm{M}+2]$ (benzene C), $117.2(\mathrm{CN}) \quad(33 \%)$

84.3, 158 (thiouracil $\mathrm{C}=\mathrm{C}), \quad 117.2(\mathrm{CN})$ $157.3(\mathrm{C}=\mathrm{N}), 182(\mathrm{C}=\mathrm{S}), \quad 290\left[\mathrm{M}^{+}\right]$ (90\%), $292[\mathrm{M}+2]$ $(32 \%)$ aliphatic),

$82.5,160.9$ (thiouracil

$\mathrm{C}=\mathrm{C}), 159.2(\mathrm{C}=\mathrm{N}), 182$

$(C=S), 122.3,128.4,131.7$,

133.9 (benzene C), 117.2

$(\mathrm{CN})$

$325\left[\mathrm{M}^{+}\right]$

$(59 \%)$,

$327\left[\mathrm{M}^{+2}\right]$

$(24 \%)$

81.1,158 (thiouracil $\mathrm{C}=\mathrm{C}$ ), $157.9(\mathrm{C}=\mathrm{N}), 182(\mathrm{C}=\mathrm{S})$, $115.1,129.2,132.4,148.5$ (benzene $\mathrm{C}), 56.3\left(3 \mathrm{CH}_{3}\right.$ aliphatic), $117(\mathrm{CN})$

$337\left[\mathrm{M}^{+}\right]$

$(100 \%)$,

$339\left[\mathrm{M}^{+2}\right]$

(33\%)

$164(\mathrm{C}=\mathrm{N}), 167.7(\mathrm{C}=\mathrm{O})$,

75, 169 (thiouracil

$\mathrm{C}=\mathrm{C}), 178(\mathrm{C}=\mathrm{S}), 115.4$,

122.1, 127, 127.1, 127.8,

128.6, 130.5, 133.2, 147.8,

161.3 ( 2 benzene C), 117

(CN)

$163(\mathrm{C}=\mathrm{N}), 165(\mathrm{C}=\mathrm{O}), 77$,

170 (thiouracil $\mathrm{C}=\mathrm{C}$ ), 178

$(\mathrm{C}=\mathrm{S}), 113,122.1,124.4$,

127, 127.1, 128.6, 143.7,

147.8 ( 2 benzene C), 43.7

$373\left[\mathrm{M}^{+}\right]$

$(51.7 \%)$

$\left(2 \mathrm{CH}_{3}\right.$ aliphatic), $117(\mathrm{CN})$

$163(\mathrm{C}=\mathrm{N}), 168(\mathrm{C}=\mathrm{O}), 75$, 170 (thiouracil $\mathrm{C}=\mathrm{C}$ ), 177 (C=S), 122.1, 122.3, 127,

$408\left[\mathrm{M}^{+}\right]$

(19.2\%),

127.1, 128.4, 128.6, 131.7,

$133.2,133.9,147.8$ (2 ben-

zene C), $117(\mathrm{CN})$

$164(\mathrm{C}=\mathrm{N}), 166(\mathrm{C}=\mathrm{O}), 77$, 169 (thiouracil $\mathrm{C}=\mathrm{C}), 175$

$(C=S), 115.1,122.1,127$,

127.1, 128.6, 129.2, 132.4, $133.2,147.8,148.5$ (2 ben-

$420\left[\mathrm{M}^{+}\right]$

$(70.1 \%)$

zene C), $117(\mathrm{CN}), 56.2$

$\left(3 \mathrm{CH}_{3}\right.$, aliphatic)

$181(\mathrm{C}=\mathrm{S}), 79.5,175.9$

( $\mathrm{C}=\mathrm{C}$ thiouracil), 168

$(\mathrm{C}=\mathrm{N}), 50\left(\mathrm{CH}_{2}\right.$ aliphatic $), \quad 286\left[\mathrm{M}^{+}\right]$

$174(\mathrm{C}=\mathrm{O}), 115.4,127.8, \quad(33 \%)$

$130.5,161.3$ (benzene $\mathrm{C}$ ), $115.7(\mathrm{CN})$ 


\begin{tabular}{|c|c|c|c|c|}
\hline $5 b$ & $\begin{array}{l}3200(\mathrm{NH}, \\
\text { stretching }) \\
2235(\mathrm{CN}) \\
1675(\mathrm{C}=\mathrm{O})\end{array}$ & $\begin{array}{l}6.9(\mathrm{~s}, 2 \mathrm{H}, \text { imidazo }), 2.5(\mathrm{~s}, \\
\left.6 \mathrm{H}, 2 \mathrm{CH}_{3}\right), 7.7-8.5(\mathrm{~m}, \\
4 \mathrm{H}, \mathrm{Ar}-\mathrm{H}), 10(\mathrm{~s}, 1 \mathrm{H},-\mathrm{NH}, \\
\left.\mathrm{D}_{2} \mathrm{O} \text { exchangeable }\right)\end{array}$ & $\begin{array}{l}183(\mathrm{C}=\mathrm{S}), 78.7,178(\mathrm{C}=\mathrm{C} \\
\text { thiouracil }), 166(\mathrm{C}=\mathrm{N}), 53.2 \\
\left(\mathrm{CH}_{2} \text { aliphatic }\right), 175 \\
(\mathrm{C}=\mathrm{O}), 113.8,124.4,127.1, \\
143.7 \text { (benzene } \mathrm{C}), 43.7 \\
\left(2 \mathrm{CH}_{3} \text { aliphatic), } 117.2\right. \\
(\mathrm{CN})\end{array}$ & $\begin{array}{l}311\left[\mathrm{M}^{+}\right] \\
(20.2 \%)\end{array}$ \\
\hline $5 c$ & $\begin{array}{l}3145(\mathrm{NH}, \\
\text { stretching) } \\
2228(\mathrm{CN}) \\
1668(\mathrm{C}=\mathrm{O})\end{array}$ & $\begin{array}{l}6.8(\mathrm{~s}, 2 \mathrm{H}, \text { imidazo }), 7-8 \\
(\mathrm{~m}, 4 \mathrm{H}, \mathrm{Ar}-\mathrm{H}), 10.2(\mathrm{~s}, 1 \mathrm{H}, \\
\left.-\mathrm{NH}, \mathrm{D}_{2} \mathrm{O} \text { exchangeable }\right)\end{array}$ & $\begin{array}{l}183(\mathrm{C}=\mathrm{S}), 79.9,172.4 \\
(\mathrm{C}=\mathrm{C} \text { thiouracil), } 164 \\
(\mathrm{C}=\mathrm{N}), 50.8\left(\mathrm{CH}_{2}\right. \\
\text { aliphatic), } 171.3(\mathrm{C}=\mathrm{O}), \\
122.3,128.4,131.7,133.9 \\
\text { (benzene } \mathrm{C}), 117(\mathrm{CN})\end{array}$ & $\begin{array}{l}346\left[\mathrm{M}^{+}\right] \\
(20.6 \%) \\
348[\mathrm{M}+2] \\
(20.7 \%)\end{array}$ \\
\hline $5 d$ & $\begin{array}{l}3162(\mathrm{NH}, \\
\text { stretching }) \\
2210(\mathrm{CN}) \\
1670(\mathrm{C}=\mathrm{O})\end{array}$ & $\begin{array}{l}3.1,3.2,3.7(\mathrm{~s}, 9 \mathrm{H}, 3 \\
\left.\mathrm{OCH}_{3}\right), 6.8(\mathrm{~s}, 2 \mathrm{H}, \\
\text { imidazo), } 7.3(\mathrm{~s}, 2 \mathrm{H}, \\
\mathrm{Ar}-\mathrm{H}), 11.2(\mathrm{~s}, 1 \mathrm{H},-\mathrm{NH}, \\
\left.\mathrm{D}_{2} \mathrm{O} \text { exchangeable }\right)\end{array}$ & $\begin{array}{l}183(\mathrm{C}=\mathrm{S}), 79.1,174(\mathrm{C}=\mathrm{C} \\
\text { thiouracil }), 167(\mathrm{C}=\mathrm{N}), 53.1 \\
\left(\mathrm{CH}_{2} \text { aliphatic }\right), 172.7 \\
(\mathrm{C}=\mathrm{O}), 115.1,129.2,132.4, \\
148.5 \text { (benzene } \mathrm{C}), 117.4 \\
(\mathrm{CN}), 56.2\left(3 \mathrm{CH}_{3}\right. \\
\text { aliphatic) }\end{array}$ & $\begin{array}{l}358\left[\mathrm{M}^{+}\right] \\
(35 \%)\end{array}$ \\
\hline $6 a$ & $\begin{array}{l}3270(\mathrm{NH}, \\
\text { stretching) } 2220 \\
(\mathrm{CN}) 1270(\mathrm{C}=\mathrm{S})\end{array}$ & $\begin{array}{l}\text { 7.4-8.4 }(\mathrm{m}, 4 \mathrm{H}, \mathrm{Ar}-\mathrm{H}) \\
10.1\left(\mathrm{~s}, 1 \mathrm{H},-\mathrm{NH}, \mathrm{D}_{2} \mathrm{O}\right. \\
\text { exchangeable) }\end{array}$ & & $\begin{array}{l}272\left[\mathrm{M}^{+}\right] \\
(65.2 \%)\end{array}$ \\
\hline $6 b$ & $\begin{array}{l}3265(\mathrm{NH}, \\
\text { stretching) } 2225 \\
(\mathrm{CN}) 1260(\mathrm{C}=\mathrm{S})\end{array}$ & $\begin{array}{l}2.5,3.5\left(\mathrm{~s}, 2 \mathrm{H}, 2 \mathrm{CH}_{3}\right) \\
7.6-7.9(\mathrm{~m}, 4 \mathrm{H}, \mathrm{Ar}-\mathrm{H}) \\
11.1\left(\mathrm{~s}, 1 \mathrm{H},-\mathrm{NH}, \mathrm{D}_{2} \mathrm{O}\right. \\
\text { exchangeable })\end{array}$ & & $\begin{array}{l}297\left[\mathrm{M}^{+}\right] \\
(70 \%)\end{array}$ \\
\hline $6 c$ & $\begin{array}{l}3250(\mathrm{NH}, \\
\text { streching) } 2230 \\
(\mathrm{CN}) 1275(\mathrm{C}=\mathrm{S})\end{array}$ & $\begin{array}{l}\text { 7-8.4 (m, } 4 \mathrm{H}, \mathrm{Ar}-\mathrm{H}), \\
10.1\left(\mathrm{~s}, 1 \mathrm{H},-\mathrm{NH}, \mathrm{D}_{2} \mathrm{O}\right. \\
\text { exchangeable })\end{array}$ & & $\begin{array}{l}332\left[\mathrm{M}^{+}\right] \\
(14.6 \%), 334 \\
{[\mathrm{M}+2](15 \%)}\end{array}$ \\
\hline $6 d$ & $\begin{array}{l}3260(\mathrm{NH}, \\
\text { streching) } \\
2215(\mathrm{CN}) \\
1265(\mathrm{C}=\mathrm{S})\end{array}$ & $\begin{array}{l}3.1,3.2,3.7(\mathrm{~s}, 9 \mathrm{H}, \\
\left.3 \mathrm{OCH}_{3}\right), 7,7.3(\mathrm{~s}, 2 \mathrm{H}, \\
\mathrm{Ar}-\mathrm{H}), 11.2(\mathrm{~s}, 1 \mathrm{H},-\mathrm{NH}, \\
\left.\mathrm{D}_{2} \mathrm{O} \text { exchangeable }\right)\end{array}$ & & $\begin{array}{l}344\left[\mathrm{M}^{+}\right] \\
(15.5 \%)\end{array}$ \\
\hline $7 a$ & $\begin{array}{l}3460-3200\left(\mathrm{NH}_{2}\right. \\
2 \mathrm{NH}) \\
2200(\mathrm{CN}) \\
1270(\mathrm{C}=\mathrm{S})\end{array}$ & $\begin{array}{l}5.1-5.7\left(\mathrm{~s}, 3 \mathrm{H}, \mathrm{NH}, \mathrm{NH}_{2}\right. \\
\left.\mathrm{D}_{2} \mathrm{O} \text { exchangeable }\right), 7.3-7.8 \\
(\mathrm{~m}, 4 \mathrm{H}, \text { Ar- } \mathrm{H}), 10.3(\mathrm{~s}, 1 \mathrm{H}, \\
\left.-\mathrm{NH}, \mathrm{D}_{2} \mathrm{O} \text { exchangeable }\right)\end{array}$ & $\begin{array}{l}\text { 84.5, } 167.1(\mathrm{C}=\mathrm{C} \text { thioura- } \\
\text { cil), } 164(\mathrm{C}=\mathrm{N}), 180(\mathrm{C}=\mathrm{S}) \\
\text { 115.4, } 127.8,130.5,161.3 \\
\text { (benzene } \mathrm{C}), 117(\mathrm{CN})\end{array}$ & $\begin{array}{l}261\left[\mathrm{M}^{+}\right] \\
(15.4 \%)\end{array}$ \\
\hline $7 b$ & $\begin{array}{l}3460-3200\left(\mathrm{NH}_{2 \prime}\right. \\
2 \mathrm{NH}) 2222(\mathrm{CN}) \\
1265(\mathrm{C}=\mathrm{S})\end{array}$ & $\begin{array}{l}2.5,3\left(\mathrm{~s}, 6 \mathrm{H}, 2 \mathrm{CH}_{3}\right), 5.3-5.7 \\
\left(\mathrm{~s}, 3 \mathrm{H}, \mathrm{NH}, \mathrm{NH} 2, \mathrm{D}_{2} \mathrm{O}\right. \\
\text { exchangeable }), 6.5-7.5 \\
(\mathrm{~m}, 4 \mathrm{H}, \mathrm{Ar}-\mathrm{H}), 10.6(\mathrm{~s}, 1 \mathrm{H}, \\
\left.-\mathrm{NH}, \mathrm{D}_{2} \mathrm{O} \text { exchangeable }\right)\end{array}$ & $\begin{array}{l}88.2,168.9(\mathrm{C}=\mathrm{C} \text { thioura- } \\
\text { cil), } 164(\mathrm{C}=\mathrm{N}), 182(\mathrm{C}=\mathrm{S}) \text {, } \\
113,124.4,127.1,143.7 \\
\text { (benzene } \mathrm{C}), 43.5\left(2 \mathrm{CH}_{3}\right. \\
\text { aliphatic }), 117(\mathrm{CN}),\end{array}$ & $\begin{array}{l}286\left[\mathrm{M}^{+}\right] \\
(44.7 \%)\end{array}$ \\
\hline $7 \mathrm{c}$ & $\begin{array}{l}3490-3264\left(\mathrm{NH}_{2}\right. \\
2 \mathrm{NH}) 2225(\mathrm{CN}) \\
1275(\mathrm{C}=\mathrm{S})\end{array}$ & $\begin{array}{l}5.2-5.7\left(\mathrm{~s}, 3 \mathrm{H}, \mathrm{NH}, \mathrm{NH}_{2}\right. \\
\left.\mathrm{D}_{2} \mathrm{O} \text { exchangeable }\right), 7.3-7.8 \\
(\mathrm{~m}, 4 \mathrm{H}, \text { Ar- } \mathrm{H}), 10.5(\mathrm{~s}, 1 \mathrm{H}, \\
\left.-\mathrm{NH}, \mathrm{D}_{2} \mathrm{O} \text { exchangeable }\right)\end{array}$ & $\begin{array}{l}87.9,169(\mathrm{C}=\mathrm{C} \text { thiouracil }) \\
164(\mathrm{C}=\mathrm{N}), 182(\mathrm{C}=\mathrm{S}) \\
\text { 122.3, } 128.4,131.7,133.9 \\
\text { (benzene } \mathrm{C}), 117(\mathrm{CN})\end{array}$ & $\begin{array}{l}321\left[\mathrm{M}^{+}\right] \\
(24.8 \%) \\
323[\mathrm{M}+2] \\
(24.7 \%)\end{array}$ \\
\hline
\end{tabular}


3490-3300 $\left(\mathrm{NH}_{2}, 3 \mathrm{OCH}_{3}\right), 5.1-5.7(\mathrm{~s}$, $1268(\mathrm{C}=\mathrm{S})$ changeable), $6.6(\mathrm{~s}, 2 \mathrm{H}$, Ar-H), 10.2 (s, 1H, -NH, $\mathrm{D}_{2} \mathrm{O}$ exchangeable)

3456-3210 (2NH, $2.4\left(\mathrm{~s}, 3 \mathrm{H}, \mathrm{COCH}_{3}\right)$, $8 a$ stretching) $2200 \quad 7.8-8.2(\mathrm{~m}, 8 \mathrm{H}, \mathrm{Ar}-\mathrm{H})$, (CN) 1690 $(\mathrm{C}=\mathrm{O})$ $10,10.5\left(\mathrm{~s}, 2 \mathrm{H}, 2 \mathrm{NH}, \mathrm{D}_{2} \mathrm{O}\right.$ exchangeable) 2NH) $2230(\mathrm{CN}) 3 \mathrm{H}, \mathrm{NH}, \mathrm{NH}_{2}, \mathrm{D}_{2} \mathrm{O}$ ex-

85.8, $167.5(\mathrm{C}=\mathrm{C}$ thiouracil), $163(\mathrm{C}=\mathrm{N}), 183(\mathrm{C}=\mathrm{S})$, $115,129.2,132.4,148.5$ (benzene $\mathrm{C}), 117(\mathrm{CN})$, $333\left[\mathrm{M}^{+}\right]$ (15.3\%)

$55.5\left(3 \mathrm{CH}_{3}\right.$ aliphatic)

88, 168.2 ( $\mathrm{C}=\mathrm{C}$ thiouracil), $164(\mathrm{C}=\mathrm{N}), 180(\mathrm{C}=\mathrm{S}), 115$, $115.1,115.5,127.4,127.8$, $129.4,130.5,151.1,161.3$ (2 benzene $\mathrm{C}), 188(\mathrm{C}=\mathrm{O})$, $364\left[\mathrm{M}^{+}\right]$ (18\%)

116.2 (CN), 22.8 (acetyl $\left.\mathrm{CH}_{3}\right)$

86, 167.3 ( $\mathrm{C}=\mathrm{C}$ thiouracil), 3450-3100 (2NH $2.3\left(\mathrm{~s}, 3 \mathrm{H}, \mathrm{COCH}_{3}\right), 2.5,3$, $3.3\left(\mathrm{~s}, 6 \mathrm{H}, 2 \mathrm{CH}_{3}\right), 7.2-8$
$(\mathrm{~m}, 8 \mathrm{H}, \mathrm{Ar}-\mathrm{H}), 10,10.4$ stretching) 2225 (CN) 1680 $(\mathrm{C}=\mathrm{O})$ (s, 2H, 2NH, $\mathrm{D}_{2} \mathrm{O}$ exchangeable)

$164(\mathrm{C}=\mathrm{N}), 180(\mathrm{C}=\mathrm{S}), 113$, 115, 24.4, 127.1, 127.4, 129.4, 143.7, 151.1 (2 benzene $\mathrm{C}), 43.2\left(2 \mathrm{CH}_{3}\right.$ $389\left[\mathrm{M}^{+}\right]$ (25\%) aliphatic), $190(\mathrm{C}=\mathrm{O}), 116.2$ (CN), 22.8 (acetyl $\mathrm{CH}_{3}$ ) 85, 166.2 ( $\mathrm{C}=\mathrm{C}$ thiouracil), 3500-3200 (NH, $2.4\left(\mathrm{~s}, 3 \mathrm{H}, \mathrm{COCH}_{3}\right), 7.5-8$ stretching) $2030 \quad(\mathrm{~m}, 8 \mathrm{H}, \mathrm{Ar}-\mathrm{H}), 10,10.3$ (CN) 1690 $(\mathrm{C}=\mathrm{O})$ (s $2 \mathrm{H}, 2 \mathrm{NH}, \mathrm{D}_{2} \mathrm{O}$ exchangeable)

$162(\mathrm{C}=\mathrm{N}), 180(\mathrm{C}=\mathrm{S}), 115$, $122.3,127.4,128.4,129.4$, 131.7, 133.9, 151.1 (2 benzene C), $190(\mathrm{C}=\mathrm{O}), 117$ (CN), 22.8 (acetyl $\mathrm{CH}_{3}$ )

$82,168.5(\mathrm{C}=\mathrm{C}$ thiouracil), 3, 3.2, $3.7\left(\mathrm{~s}, 9 \mathrm{H}, 3 \mathrm{OCH}_{3}\right), 163(\mathrm{C}=\mathrm{N}), 180(\mathrm{C}=\mathrm{S}), 110$, $2.3\left(\mathrm{~s}, 3 \mathrm{H}, \mathrm{COCH}_{3}\right), 7.2-8 \quad 115,127.4,129.2,129.4$,

3550-3300 (NH $8 d$ stretching) 2230 (CN) 1690 $(\mathrm{C}=\mathrm{O})$ (m, 6H, Ar-H), 10, 10.5 (s, $2 \mathrm{H}, 2 \mathrm{NH}, \mathrm{D}_{2} \mathrm{O}$ exchangeable)

3480-3300 (2NH, 1.9 (s, 3H, $\left.-\mathrm{CH}_{3}\right), 2.5$

$9 a$ stretching) $2200\left(\mathrm{~s}, 3 \mathrm{H}, \mathrm{N}-\mathrm{CH}_{3}\right), 7-7.8(\mathrm{~m}$, (CN) 1690 9H, Ar-H), 10, 10.5 (s, 2H, $(\mathrm{C}=\mathrm{O})$ $2 \mathrm{NH}, \mathrm{D}_{2} \mathrm{O}$ exchangeable)

$2.2\left(\mathrm{~s}, 3 \mathrm{H},-\mathrm{CH}_{3}\right), 2.5$

3470-3200 (2NH, stretching) 2225 (CN) 1680 $(\mathrm{C}=\mathrm{O})$ $\left(\mathrm{s}, 3 \mathrm{H}, \mathrm{N}-\mathrm{CH}_{3}\right), 3.1-3.4$ $\left(\mathrm{s}, 6 \mathrm{H}, 2 \mathrm{CH}_{3}\right), 7.4-7.9(\mathrm{~m}$, 9H, Ar-H), 10.2, 11 (s, 2H, $2 \mathrm{NH}, \mathrm{D}_{2} \mathrm{O}$ exchangeable)
$132.4,148.5,151.1$ (2 ben-

zene C), $190(\mathrm{C}=\mathrm{O}), 117$

(CN), 22.5 (acetyl $\mathrm{CH}_{3}$ ),

$55.5\left(3 \mathrm{CH}_{3}\right.$ aliphatic)

$84,166(\mathrm{C}=\mathrm{C}$ thiouracil), $164(\mathrm{C}=\mathrm{N}), 182(\mathrm{C}=\mathrm{S}), 116$, 133.5 ( $\mathrm{C}=\mathrm{C}$ antipyrene), $160(\mathrm{C}=\mathrm{O}), 112,115.4, \quad 432\left[\mathrm{M}^{+}\right]$ $118.9,127.8,129,130.5$, 15,35 (2 $\mathrm{CH}_{3}$ aliphatic), $117(\mathrm{CN})$

85.2, 168 ( $\mathrm{C}=\mathrm{C}$ thiouracil), $164(\mathrm{C}=\mathrm{N}), 182(\mathrm{C}=\mathrm{S})$, 116.2, $133.6(\mathrm{C}=\mathrm{C}$ antipyrene), $161(\mathrm{C}=\mathrm{O}), 112, \quad 457\left[\mathrm{M}^{+}\right]$ $113,118.9,124.4,127.1, \quad(14.9 \%)$ $129,142.2,143.7$ ( 2 benzene C), 14.9, 35.3, 43 (4 $\mathrm{CH}_{3}$ aliphatic), $117.4(\mathrm{CN})$ $142.2,161$ ( 2 benzene C),
$424\left[\mathrm{M}^{+}\right]$

(14.2\%), 426 [M+2] (14.3\%)

$436\left[\mathrm{M}^{+}\right]$

(9.8\%)

(12.2\%) 


\begin{tabular}{|c|c|c|c|c|}
\hline $9 c$ & $\begin{array}{l}3480-3210(2 \mathrm{NH}, \\
\text { stretching }) 2215 \\
(\mathrm{CN}) 1690 \\
(\mathrm{C}=\mathrm{O})\end{array}$ & $\begin{array}{l}2\left(\mathrm{~s}, 3 \mathrm{H},-\mathrm{CH}_{3}\right), 2.5(\mathrm{~s}, 3 \mathrm{H}, \\
\left.\mathrm{N}-\mathrm{CH}_{3}\right), 6.8-7.8(\mathrm{~m}, 9 \mathrm{H}, \\
\mathrm{Ar}-\mathrm{H}), 10.1,10.6(\mathrm{~s}, 2 \mathrm{H}, \\
\left.2 \mathrm{NH}, \mathrm{D}_{2} \mathrm{O} \text { exchangeable }\right)\end{array}$ & $\begin{array}{l}\text { 85.4, } 168.3(\mathrm{C}=\mathrm{C} \text { thioura- } \\
\text { cil), } 164(\mathrm{C}=\mathrm{N}), 184(\mathrm{C}=\mathrm{S}), \\
\text { 116.6, } 135(\mathrm{C}=\mathrm{C} \text { antipy- } \\
\text { rene } 160.7(\mathrm{C}=\mathrm{O}), 112, \\
118.9,122.3,128.4,129, \\
\text { 131.7, 133.9, 142.2 }(2 \mathrm{ben}- \\
\text { zene } \mathrm{C}), 14.9,35.5\left(2 \mathrm{CH}_{3}\right. \\
\text { aliphatic), } 117.2(\mathrm{CN})\end{array}$ & $\begin{array}{l}494\left[\mathrm{M}^{+}\right] \\
(12.3 \%) \\
492[\mathrm{M}+2] \\
(12.5 \%)\end{array}$ \\
\hline $9 d$ & $\begin{array}{l}3460-3250(2 \mathrm{NH}, \\
\text { stretching }) 2225 \\
(\mathrm{CN}) 1690 \\
(\mathrm{C}=\mathrm{O})\end{array}$ & $\begin{array}{l}2.2\left(\mathrm{~s}, 3 \mathrm{H},-\mathrm{CH}_{3}\right), 2.5(\mathrm{~s}, \\
\left.3 \mathrm{H}, \mathrm{N}-\mathrm{CH}_{3}\right), 3.1-3.4(\mathrm{~s}, \\
\left.9 \mathrm{H}, \mathrm{OCH}_{3}\right), 7-.5(\mathrm{~m}, 7 \mathrm{H}, \\
\mathrm{Ar}-\mathrm{H}), 9.5,11(\mathrm{~s}, 2 \mathrm{H}, 2 \mathrm{NH}, \\
\left.\mathrm{D}_{2} \mathrm{O} \text { exchangeable }\right)\end{array}$ & $\begin{array}{l}\text { 85.5, } 167.6(\mathrm{C}=\mathrm{C} \text { thioura- } \\
\text { cil), } 164(\mathrm{C}=\mathrm{N}), 182(\mathrm{C}=\mathrm{S}), \\
116.3,133.7(\mathrm{C}=\mathrm{C} \text { antipy- } \\
\text { rene }), 160.7(\mathrm{C}=\mathrm{O}), 112, \\
115,118.9,129,129.2, \\
132.4,142.2,148.5(2 \text { ben- } \\
\text { zene } \mathrm{C}), 14.9,35.2,55.6(5 \\
\left.\mathrm{CH}_{3} \text { aliphatic }\right), 117.9(\mathrm{CN})\end{array}$ & $\begin{array}{l}504\left[\mathrm{M}^{+}\right] \\
(11.4 \%)\end{array}$ \\
\hline $10 \mathrm{a}$ & $\begin{array}{l}3495-3300(2 \mathrm{NH}, \\
\text { stretching }) 2200 \\
(\mathrm{CN}) 1700 \\
(\mathrm{C}=\mathrm{O})\end{array}$ & $\begin{array}{l}7.8-8.2(\mathrm{~m}, 8 \mathrm{H}, \mathrm{Ar}-\mathrm{H}) \\
10,10.4\left(\mathrm{~s}, 2 \mathrm{H}, 2 \mathrm{NH}, \mathrm{D}_{2} \mathrm{O}\right. \\
\text { exchangeable }), 12(\mathrm{~s}, 1 \mathrm{H}, \\
\left.\mathrm{COOH}, \mathrm{D}_{2} \mathrm{O} \text { exchangable }\right)\end{array}$ & $\begin{array}{l}90,166(\mathrm{C}=\mathrm{C} \text { thiouracil), } \\
162(\mathrm{C}=\mathrm{N}), 178(\mathrm{C}=\mathrm{S}), 115, \\
115.4,117.2,118.4,127.8, \\
130.5,130.9,134.5,148.3, \\
161.3(2 \text { benzene } \mathrm{C}), 117.9 \\
(\mathrm{CN}), 170 \text { (Carboxyl C) }\end{array}$ & $\begin{array}{l}366\left[\mathrm{M}^{+}\right] \\
(62 \%)\end{array}$ \\
\hline $10 \mathrm{~b}$ & $\begin{array}{l}3468-3100(\mathrm{NH}, \\
\text { stretching }) 2225 \\
(\mathrm{CN}) 1710 \\
(\mathrm{C}=\mathrm{O})\end{array}$ & $\begin{array}{l}2.3,2.5\left(\mathrm{~s}, 6 \mathrm{H}, 2 \mathrm{CH}_{3}\right), \\
\text { 7.2-8 }(\mathrm{m}, 8 \mathrm{H}, \mathrm{Ar}-\mathrm{H}), \\
\text { 10,10.4 }\left(\mathrm{s}, 2 \mathrm{H}, 2 \mathrm{NH}, \mathrm{D}_{2} \mathrm{O}\right. \\
\text { exchangeable }), 12(\mathrm{~s}, 1 \mathrm{H}, \\
\text { COOH, } \mathrm{D}_{2} \mathrm{O} \text { exchange- } \\
\text { able) }\end{array}$ & $\begin{array}{l}90,167(\mathrm{C}=\mathrm{C} \text { thiouracil), } \\
161(\mathrm{C}=\mathrm{N}), 179(\mathrm{C}=\mathrm{S}), 113, \\
115,117,118.4,124.4, \\
127.1,130.9,134.5,143.7 \\
148.3,(2 \text { benzene } \mathrm{C}), 117.9 \\
(\mathrm{CN}), 43.2\left(2 \mathrm{CH}_{3} \text { alipha- }\right. \\
\text { tic), } 171 \text { (Carboxyl } \mathrm{C})\end{array}$ & $\begin{array}{l}391\left[\mathrm{M}^{+}\right] \\
(58 \%)\end{array}$ \\
\hline $10 \mathrm{c}$ & $\begin{array}{l}3485-3200(\mathrm{NH}, \\
\text { stretching }) 2250 \\
(\mathrm{CN}) 1730 \\
(\mathrm{C}=\mathrm{O})\end{array}$ & $\begin{array}{l}7.5-8.2(\mathrm{~m}, 8 \mathrm{H}, \mathrm{Ar}-\mathrm{H}), \\
10,10.5(\mathrm{~s}, 2 \mathrm{H}, 2 \mathrm{NH}, \\
\left.\mathrm{D}_{2} \mathrm{O} \text { exchangeable }\right), 12 \\
\left(\mathrm{~s}, 1 \mathrm{H}, \mathrm{COOH}, \mathrm{D}_{2} \mathrm{O}\right. \\
\text { exchangeable) }\end{array}$ & $\begin{array}{l}88,164(\mathrm{C}=\mathrm{C} \text { thiouracil), } \\
162(\mathrm{C}=\mathrm{N}), 179(\mathrm{C}=\mathrm{S}) \\
115,116,117,118.4,122.3 \\
128.4,131.7,133.9,134.5 \\
148.3(2 \text { benzene } \mathrm{C}), 117.9 \\
(\mathrm{CN}), 170 \text { (Carboxyl C) }\end{array}$ & $\begin{array}{l}426\left[\mathrm{M}^{+}\right] \\
(55.1 \%) \\
428[\mathrm{M}+2] \\
(55.2 \%)\end{array}$ \\
\hline $10 \mathrm{~d}$ & $\begin{array}{l}3469-3190(\mathrm{NH}, \\
\text { stretching) } 2200 \\
(\mathrm{CN}) 1720 \\
(\mathrm{C}=\mathrm{O})\end{array}$ & $\begin{array}{l}3,3.2,3.7\left(9 \mathrm{H}, 3 \mathrm{OCH}_{3}\right), \\
7-8(\mathrm{~m}, 6 \mathrm{H}, \mathrm{Ar}-\mathrm{H}), 10, \\
10.4\left(\mathrm{~s}, 2 \mathrm{H}, 2 \mathrm{NH}, \mathrm{D}_{2} \mathrm{O}\right. \\
\text { exchangeable }), 12(\mathrm{~s}, \\
1 \mathrm{H}, \mathrm{COOH}, \mathrm{D}_{2} \mathrm{O} \\
\text { exchangeable })\end{array}$ & $\begin{array}{l}\text { 90, } 169(\mathrm{C}=\mathrm{C} \text { thiouracil), } \\
162(\mathrm{C}=\mathrm{N}), 179(\mathrm{C}=\mathrm{S}), 115, \\
129.2,132.4,148 \text { (benzene } \\
\mathrm{C}), 117(\mathrm{CN}), 170.5 \\
\text { (Carboxyl C), } 55.5\left(3 \mathrm{CH}_{3}\right. \\
\text { aliphatic) }\end{array}$ & $\begin{array}{l}438\left[\mathrm{M}^{+}\right] \\
(60 \%)\end{array}$ \\
\hline
\end{tabular}

\section{Syntheses}

6-Aryl-4-oxo-2-thioxo-1,2,3,4-tetrahydropyrimidine-5-carbonitriles (1a-d). - Synthesis of compounds 1a-d was performed according to the literature (13). A mixture of ethyl cyanoacetate $(11.3 \mathrm{~mL}, 0.1 \mathrm{~mol})$, thiourea $(7.6 \mathrm{~g}, 0.1 \mathrm{~mol})$ and the appropriate aldehyde [ $p$-fluorobenzaldehyde $(1.24 \mathrm{~g}, 0.1 \mathrm{~mol}), p$-dimethylamino benzaldehyde $(1.49 \mathrm{~g}, 0.1 \mathrm{~mol})$, $p$-bromobenzaldehyde $(1.85 \mathrm{~g}, 0.1 \mathrm{~mol})$ and 3,4,5-trimethoxy benzaldehyde $(1.96 \mathrm{~g}, 0.1$ 
mol)] in $50 \mathrm{~mL}$ sodium ethoxide $(2.3 \mathrm{~g}, 0.1 \mathrm{~mol})$ was stirred for $24 \mathrm{~h}$ at room temperature and poured onto ice-water, acidified with dil. $\mathrm{HCl}$ to give precipitates, which were filtered, dried and recrystallized from DMF/water to give compounds 1a-d, respectively.

6-Aryl-4-oxo-2-thioxo-1,2,3,4-tetrahydropyrimidine-5-carboxamides (2a-d). - The appropriate thiouracil derivative, 1a $(2.47 \mathrm{~g}, 0.01 \mathrm{~mol}), 1 \mathbf{b}(2.72 \mathrm{~g}, 0.01 \mathrm{~mol}), 1 \mathrm{c}(3.08 \mathrm{~g}, 0.01$ $\mathrm{mol})$ or $1 \mathrm{~d}(3.19 \mathrm{~g}, 0.01 \mathrm{~mol})$ in conc. sulphuric acid $(40 \mathrm{~mL})$, was refluxed for $3 \mathrm{~h}$, cooled, poured onto ice-water and neutralized with ammonia to give compounds $\mathbf{2 a - d}$, respectively, in the form of precipitates, which were filtered, dried and recrystallized from DMF/ water.

6-Aryl-4-chloro-2-thioxo-1,2-dihydropyrimidine-5-carbonitriles (3a-d). - The appropriate thiouracil derivative, $1 \mathbf{a}(2.47 \mathrm{~g}, 0.01 \mathrm{~mol}), \mathbf{1 b}(2.72 \mathrm{~g}, 0.01 \mathrm{~mol}), 1 \mathrm{c}(3.08 \mathrm{~g}, 0.01 \mathrm{~mol})$ or 1d $(3.19 \mathrm{~g}, 0.01 \mathrm{~mol})$, was heated under reflux in phosphorus oxychloride $(25 \mathrm{~mL})$ and phosphorus pentachloride $(2 \mathrm{~g}, 0.01 \mathrm{~mol})$ for $8 \mathrm{~h}$, cooled and poured onto ice to precipitate compounds $3 \mathbf{a}-\mathbf{d}$, which were washed with petroleum ether, filtered off and dried under vacuum.

3-Aryl-10-oxo-1-thioxo-2,10-dihydro-1H-pyrimido[6,1-b]quinazoline-4-carbonitriles (4a-d). - A mixture of the appropriate thiouracil, 3a (2.65 g, $0.01 \mathrm{~mol}), 3 \mathrm{~b}(2.90 \mathrm{~g}, 0.01 \mathrm{~mol}), 3 \mathrm{c}$ $(3.26 \mathrm{~g}, 0.01 \mathrm{~mol})$ or $3 \mathrm{~d}(3.37 \mathrm{~g}, 0.01 \mathrm{~mol})$, and anthranilic acid $(1.37 \mathrm{~g}, 0.01 \mathrm{~mol})$ was refluxed in butanol $(50 \mathrm{~mL})$ for $10-12 \mathrm{~h}$, cooled, filtered, dried and recrystallized from $\mathrm{DMF} /$ water to give compounds $4 \mathbf{a}-\mathbf{d}$, respectively.

7-Aryl-3-oxo-5-thioxo-2,3,5,6-tetrahydroimidazo[1,2-c]pyrimidine-8-carbonitriles (5a-d). A mixture of the appropriate thiouracil, 3a $(2.65 \mathrm{~g}, 0.01 \mathrm{~mol}), 3 \mathrm{~b}(2.90 \mathrm{~g}, 0.01 \mathrm{~mol}), 3 \mathrm{c}$ $(3.26 \mathrm{~g}, 0.01 \mathrm{~mol})$ or $3 \mathrm{~d}(3.37 \mathrm{~g}, 0.01 \mathrm{~mol})$ and glycine $(0.75 \mathrm{~g}, 0.01 \mathrm{~mol})$, was refluxed in butanol (30 mL) for 6-9 h, cooled and the separated solid was refluxed with acetic anhydride $(10 \mathrm{~mL})$ for $3 \mathrm{~h}$, cooled, filtered, dried and recrystallized from DMF/water to yield compounds 5a-d, respectively.

7-Aryl-5-thioxo-5,6-dihydrotetrazolo[1,5-c]pyrimidine-8-carbonitriles (6a-d). - A mixture of the appropriate thiouracil, $3 \mathrm{a}(2.65 \mathrm{~g}, 0.01 \mathrm{~mol}), 3 \mathbf{b}(2.90 \mathrm{~g}, 0.01 \mathrm{~mol}), 3 \mathrm{c}(3.26 \mathrm{~g}, 0.01$ $\mathrm{mol})$ or $3 \mathrm{~d}(3.37 \mathrm{~g}, 0.01 \mathrm{~mol})$ and sodium azide $(0.65 \mathrm{~g}, 0.01 \mathrm{~mol})$, was refluxed in glacial acetic acid $(30 \mathrm{~mL})$ for $5-8 \mathrm{~h}$, cooled and the solid was filtered, dried and recrystallized from DMF/water to give compounds $6 \mathbf{a}-\mathbf{d}$, respectively.

6-Aryl-4-hydrazino-2-thioxo-1,2-dihydropyrimidine-5-carbonitriles (7a-d). - A mixture of the appropriate thiouracil, $3 \mathbf{a}(2.65 \mathrm{~g}, 0.01 \mathrm{~mol}), 3 \mathbf{b}(2.90 \mathrm{~g}, 0.01 \mathrm{~mol}), 3 \mathrm{c}(3.26 \mathrm{~g}, 0.01 \mathrm{~mol})$ or $3 \mathbf{d}(3.37 \mathrm{~g}, 0.01 \mathrm{~mol})$ and hydrazine hydrate $(20 \mathrm{~mL}, 99 \%)$, was refluxed in methanol $(30 \mathrm{~mL})$ for $20 \mathrm{~min}$, cooled, stirred for $24 \mathrm{~h}$ and poured onto ice-water. The solid obtained was filtered, dried and recrystallized from DMF/water to yield compounds 7a-d, respectively.

4-(p-Acetyl-phenylamino)-6-aryl-2-thioxo-1,2-dihydropyrimidine-5-carbonitrile ( $8 a$-d). - A mixture of the appropriate thiouracil, $3 \mathrm{a}(1.32 \mathrm{~g}, 0.05 \mathrm{~mol}), 3 \mathrm{~b}(1.45 \mathrm{~g}, 0.05 \mathrm{~mol}), 3 \mathrm{c}(1.36$ $\mathrm{g}, 0.05 \mathrm{~mol})$ or $3 \mathrm{~d}(1.68 \mathrm{~g}, 0.05 \mathrm{~mol})$ and $p$-aminoacetophenone $(0.67 \mathrm{~g}, 0.05 \mathrm{~mol})$, was refluxed in $25 \mathrm{~mL}$ anhydrous pyridine for $10 \mathrm{~h}$, cooled, poured onto ice- $\mathrm{HCl}$, filtered, dried and recrystallized from ethyl acetate to give compounds 8a-d, respectively. 
4-(1,5-Dimethyl-3-oxo-2-phenyl-2,3-dihydro-1H-pyrazol-4-ylamino)-6-aryl-2-thioxo-1,2-dihydropyrimidine-5-carbonitrile $(\mathbf{9} a-d)$ and 2-[5-cyano-6-aryl-2-thioxo-1,2-dihydropyrimidine-4-yl-amino]-benzoic acid $(\mathbf{1 0} a-d)$. General procedure. - A mixture of the appropriate thiouracil, 3a (1.325 g, $0.05 \mathrm{~mol}), 3 \mathrm{~b}(1.45 \mathrm{~g}, 0.05 \mathrm{~mol}), 3 \mathrm{c}(1.36 \mathrm{~g}, 0.05 \mathrm{~mol})$ or 3d $(1.68 \mathrm{~g}, 0.05$ $\mathrm{mol})$ and 4-amino-1,5-dimethyl-2-phenyl-1,2-dihydro-3H-pyrazol-3-one (4-aminoantipyrine) $(1 \mathrm{~g}, 0.05 \mathrm{~mol})$ or anthranilic acid $(0.68 \mathrm{~g}, 0.05 \mathrm{~mol})$, was refluxed in $25 \mathrm{~mL}$ methanol containing $1 \mathrm{~mL}$ anhydrous pyridine for $12 \mathrm{~h}$, cooled, poured onto ice- $\mathrm{HCl}$, filtered, dried and recrystallized from DMF/water to give $\mathbf{9 a - d}$ or $\mathbf{1 0 a - d}$, respectively.

\section{Antimicrobial activity}

All compounds were evaluated for antibacterial activity against Bacillus subtilis (ATCC 6051), Staphylococcus aureus (ATCC 12600) (Gram-positive), Escherichia coli (ATCC 11775) (Gram-negative) and Candida albicans (ATCC 26555) and Aspergillus flavus (ATCC-11495) (fungi) microorganisms using the disc diffusion method. Disc diffusion sensitivity test was done in the manner identical to that of Bauer et al. (14). All microorganisms used were obtained from the culture collection of the Department of Microbiology, Microanalytical Centre, Faculty of Science, Cairo University, Cairo, Egypt.

Media for disc sensitivity tests were nutrient agar and Müller-Hinton agar (MHA) purchased from Difco (USA). Non-sterile powder of tested compounds and penicillin and nystatin standards were dissolved in sterile DMSO to yield $2.0 \mu \mathrm{g} \mathrm{mL}-1$ solution, and passed through a $0.2-\mu \mathrm{m}$ membrane filter (Millipore Corp, USA). Penicillin (Bioanalyse, Turkey) and nystatin (Sigma-Aldrich, USA) were used as reference substances. Inhibition zones were measured in millimeters at the end of an incubation period of $48 \mathrm{~h}$ at $28^{\circ} \mathrm{C}$.

\section{RESULTS AND DISCUSSION}

\section{Chemistry}

A series of 6-aryl-5-cyano-2-thiouracils (1a-d) were newly synthesized in a facile manner from thiourea, ethyl cyanoacetate and different aldehydes in sodium ethoxide instead of anhydrous $\mathrm{K}_{2} \mathrm{CO}_{3}$ / ethanol (14) with better yield. 6-Aryl-5-carboxamide-2-thioxo-2,3-dihydropyrrimidine-4- $(1 H)$-ones $(2 \mathrm{a}-\mathbf{d})$ were obtained by the reaction of thiouracils 1a-d with concentrated sulphuric acid. Thiouracils 1a-d were converted to 4-chloropyrimidines 3a-d by refluxing with a mixture of phosphorus oxychloride/phosphorus pentachloride (15). The chloropyrimidine derivatives 3a-d were used as the starting material for preparation of new heterocyclic compounds. Thus, 3a-d reacted with anthranilic acid, glycine, sodium azide or hydrazine hydrate to yield 10-oxo-3-aryl-1-thioxo2,10-dihydro- $1 H$-pyrimido[6,1-b]quinazoline-4-carbonitriles (4a-d), 7-aryl-3-oxo-5-thioxo2,3,5,6-tetrahydroimidazo[1,2-c] pyrimidine-8-carbonitriles (5a-d), 7-aryl-5-thioxo-5,6-dihydrotetrazolo[1,5-c]pyrimidine-8-carbonitriles (6a-d) and 6-aryl-4-hydrazino-2-thioxo-1,2-dihydropyrimidine-5-carbonitriles (7a-d), respectively. Reaction of 3a-d with 4-aminoacetophenone in anhydrous pyridine gave (4-acetyl-phenylamino)-6-aryl-2-thioxo-1,2-dihydropyrimidine-5-carbonitriles (8a-d). Finally, 4-amino pyrimidines $\mathbf{9 a - d}$ and $10 \mathbf{a}-\mathbf{d}$ were obtained by the reaction of 3a-d with amines, namely, 4-amino-1,5-dimethyl-2-phe- 
nyl-1,2-dihydro-3H-pyrazol-3-one (4-aminoantipyrine) or anthranilic acid, respectively, in absolute ethanol containing pyridine. The structures of new compounds were confirmed by MS, IR, ${ }^{1} \mathrm{H}$ NMR and ${ }^{13} \mathrm{C}$ NMR as well as elemental analysis. IR spectra of compounds 1a-d showed CN bands at 2200-2235 $\mathrm{cm}^{-1}$ and a stretching $\mathrm{NH}$ band at 3450-3000 $\mathrm{cm}^{-1}$. The ${ }^{13} \mathrm{C}$ NMR spectra displayed signals for $\mathrm{C}=\mathrm{O}$ carbon and for $\mathrm{CN}$ carbon at position $\mathrm{C}-5$ of the molecule. The rest of the spectrum was in good agreement with the structures. The recorded IR spectra of compounds $\mathbf{2 a - d}$ showed the absence of $\mathrm{CN}$ as well as the presence of amide carbonyl at $1660 \mathrm{~cm}^{-1}$ and ${ }^{1} \mathrm{H}$ NMR showed a singlet signal for $\mathrm{CONH}_{2}$ proton at $5.1 \mathrm{ppm}$. Moreover, the ${ }^{13} \mathrm{C}$ NMR spectrum showed the presence of two $\mathrm{C}=\mathrm{O}$ signals corresponding to $\mathrm{C}=\mathrm{O}$ of thiouracil and amide. Also, the structures of 3a-d were established on the basis of IR, which showed the absence of $\mathrm{C}=\mathrm{O}$, while ${ }^{1} \mathrm{H}$ NMR spectra revealed a signal for $\mathrm{NH}$ proton instead of two signals like in 2a-d. Their mass spectra gave the characteristic fragmentation pattern due to the presence of chlorine atoms in the compounds. The IR spectra of compounds $4 a-d$ showed the presence of $\mathrm{C}=\mathrm{O}$ group (Table II). The ${ }^{1} \mathrm{H}$ and ${ }^{13} \mathrm{C}$ NMR spectra of products $4 \mathrm{a}-\mathrm{d}$ were compatible with the proposed structure. The IR spectra of compounds $\mathbf{5 a - d}$ showed the presence of $\mathrm{C}=\mathrm{O}$ group; their ${ }^{13} \mathrm{C}$ NMR spectrum displayed signals for $\mathrm{C}=\mathrm{O}$ carbon and for $\mathrm{CH}_{2}$ aliphatic carbon. The rest of the spectrum was in good agreement with the structures. Compounds $6 \mathbf{a}-\mathbf{d}$ and $7 \mathbf{a}-\mathbf{d}$ were confirmed by spectral data (Table II) and the mass spectrum studies of these compounds gave additional evidence for the proposed structures. ${ }^{1} \mathrm{H}$ NMR for 8a-d showed singlet signals for $\mathrm{COCH}_{3}$ and two $\mathrm{NH}$, for $\mathbf{9 a}-\mathbf{d}$ signals for $\mathrm{CH}_{3}, \mathrm{~N}-\mathrm{CH}_{3}$ and two $\mathrm{NH}$, for 10a-d signals for two $\mathrm{NH}$ ) and COOH. Also, MS spectra gave their molecular ion peaks (Table II). The ${ }^{13} \mathrm{C}$ NMR spectra for compounds 8-10 were compatible with the proposed structures.

\section{Antimicrobial activity}

The data presented in Table III showed that compounds $2 \mathbf{a}-\mathbf{d}$ and $7 \mathbf{a}-\mathbf{d}$ possess a pronounced antimicrobial activity against Staphylococcus aureus, Bacillus subtilis and Escherichia coli compared to the reference drug penicillin. As far as antifungal activity is concerned, compounds $7 \mathbf{a}-\mathbf{d}$ exhibited even stronger activity than nystatin against Candida albicans and compounds $\mathbf{2 a}, \mathbf{2 d}, \mathbf{3 a}, \mathbf{7 a}$ and $\mathbf{7 d}$ against Aspergillus flavus. Compounds 1, 2 and 3a-d showed moderate activity against the fungus Candida albicans and compounds $4 \mathbf{a}$ and $\mathbf{6 d}$ displayed moderate activity against the fungus Aspergillus flavus. Compounds 9, 10a-d, 1, 3a-d, 4c, 5a,d and 6a,c were either inactive or moderately to fairly active against the tested bacteria. To sum up all tested compounds have moderate to high antibacterial activity, except compounds $\mathbf{9 a - d}$ and $\mathbf{1 0 a - d}$, which are inactive. Compounds $\mathbf{2 a}, \mathbf{c}$ and $\mathbf{7 a}$ showed the highest antibacterial activity whereas compounds $\mathbf{7 a}, \mathbf{d}$ exhibited good to excellent results against Candida albicans and Aspergillus flavus. The present study revealed that conversion of cyano group at 5 '-position to amide (2a-d) caused a pronounced inhibition effect against Gram-positive (Staphylococcus aureus, Bacillus subtilis) and Gram-negative (Escherichia coli) bacteria. The same effects were observed after transformation of $\mathrm{Cl}$ group into $\mathrm{NHNH}_{2}(7 \mathbf{a}-\mathbf{d})$. On the other hand, increased antibacterial activity was achieved by incorporation of chloro moiety as in 4-chloro-6-(4-fluorophenyl)-2-thioxo-1,2,3,4-tetrahydropyrimidine-5-carbonitrile (3a). However, cyclization to pyrimidoquinazolines, imidazopyrimidines, tetrapyrimidines, or incorporation of 4-aminoacetophenone as in 10-oxo-1-thioxo-3-(3,4,5-trimethoxyphenyl)-2,10-dihydro- $1 H$-yrimido[6,1- $b$ ] 
M. S. Mohamed et al.: Synthesis and antimicrobial evaluation of some 6-aryl-5-cyano-2-thiouracil derivatives, Acta Pharm. 61 (2011) $171-185$.

Table III. Antimicrobial activity of the synthesized compounds

\begin{tabular}{|c|c|c|c|c|c|}
\hline \multirow{2}{*}{ Compd. $^{\mathrm{a}}$} & \multicolumn{5}{|c|}{ Inhibition zone diameter (mm) } \\
\hline & S. aureus & B. subtilis & E. coli & C. albicans & A. flavus \\
\hline 1a & 14 & 10 & 14 & 13 & - \\
\hline $1 b$ & 13 & 9 & 12 & 12 & - \\
\hline 1c & 15 & 12 & 14 & 14 & - \\
\hline $1 d$ & 13 & 10 & 13 & 13 & - \\
\hline $2 a$ & 39 & 25 & 38 & 14 & 18 \\
\hline $2 b$ & 30 & 23 & 30 & 13 & - \\
\hline $2 c$ & 35 & 24 & 34 & 15 & - \\
\hline $2 d$ & 30 & 22 & 30 & 16 & 22 \\
\hline $3 a$ & 18 & 14 & 18 & 16 & 17 \\
\hline $3 b$ & 16 & 12 & 14 & 14 & - \\
\hline $3 c$ & 17 & 13 & 15 & 15 & - \\
\hline $3 d$ & 15 & 14 & 14 & 14 & - \\
\hline $4 a$ & 20 & 17 & 19 & - & 11 \\
\hline $4 b$ & 20 & 16 & 21 & - & - \\
\hline $4 c$ & 18 & 18 & 16 & - & - \\
\hline $4 d$ & 22 & 21 & 23 & - & - \\
\hline $5 a$ & 18 & 19 & 19 & - & - \\
\hline $5 b$ & 24 & 15 & 21 & - & - \\
\hline $5 c$ & 20 & 16 & 16 & - & - \\
\hline $5 d$ & 16 & 15 & 25 & - & - \\
\hline $6 a$ & 19 & 15 & 21 & - & - \\
\hline $6 b$ & 22 & 16 & 22 & - & - \\
\hline $6 c$ & 18 & 17 & 20 & - & - \\
\hline $6 \mathrm{~d}$ & 25 & 18 & 22 & - & 14 \\
\hline $7 \mathbf{a}$ & 30 & 26 & 28 & 28 & 44 \\
\hline $7 \mathbf{b}$ & 26 & 28 & 30 & 23 & - \\
\hline $7 c$ & 28 & 25 & 27 & 27 & - \\
\hline $7 d$ & 27 & 30 & 33 & 24 & 29 \\
\hline $8 a$ & 23 & 14 & 27 & - & - \\
\hline $8 b$ & 23 & 12 & 25 & - & \\
\hline $8 c$ & 22 & 13 & 26 & - & - \\
\hline $8 d$ & 24 & 12 & 24 & - & - \\
\hline Penicillin $^{b}$ & 20 & 14 & 12 & - & - \\
\hline Nystatin $^{\mathrm{b}}$ & - & - & - & 17 & 15 \\
\hline
\end{tabular}

- No inhibition.

a $2.0 \mu \mathrm{g} \mathrm{mL}^{-1}$ in DMSO.

DMSO shows no activity. 
quinazoline-4-carbonitrile (4d), 7-[4-(dimethylamino)phenyl]-3-oxo-5-thioxo-2,3,5,6-tetrahydroimidazo[1,2-c]pyrimidine-8-carbonitrile (5b), 7-[4-dimethylamino)phenyl]-5,6-dihydrotetrazolo[1,5-c]pyrimidine-8-carbonitrile (6b), 5-thioxo-7-(3,4,5-trimethoxyphenyl)-5,6-dihydrotetrazolo[1,5-c]pyrimidine-8-arbonitrile (6d) and 4-[(4-acetylphenyl)amino]-6-(4-fluorophenyl)-2-thioxo-1,2-dihydropyrimidine-5-carbonitrile (8a) increased antibacterial activity. However, incorporation of 4-amino-1,5-dimethyl-2-phenyl-1,2-dihydro-3H-pyrazol-3-one (4-aminoantipyrine) or anthranilic acid ring to the thiouracil derivatives as in 9, 10a-d diminished antimicrobial activity. The structure activity relationship suggested that thiouracils containing amide or hydrazine hydrate moiety showed higher antibacterial and antifungal activities than other derivatives.

\section{CONCLUSIONS}

Antimicrobial studies have revealed that the most promising compounds are 6-(4-fluorophenyl)-4-oxo-2-thioxo-1,2,3,4-tetrahydropyrimidine-5-carboxamide (2a), 4-oxo-2-thioxo-6-(3,4,5-trimethoxyphenyl)-1,2,3,4-tetrahydropyrimidine-5-carboxamide (2d), 6-(4-fluorophenyl)-4-hydrazino-2-thioxo-1,2-dihydropyrimidine-5-cabonitrile (7a) and 4-hydrazino-2-thioxo-6-(3,4,5-trimethoxyphenyl)-1,2-dihydropyrimidine-5-carbonitrile (7d). Based on the above studies, the promising compounds can be submitted to in vivo antimicrobial studies as a future perspective.

\section{REFERENCES}

1. S. H. Bantawal, M. Manjathuru, K. S. Mari and K. M. Padiyath, Synthesis of some novel pyrazolo[3,4- $d]$ pyrimidine derivatives as potential antimicrobial agents, Bioorg. Med. Chem. 14 (2006) 2040-2047; DOI: 10.1016/j.bmc. 2005.10.053.

2. C. Mugnaini, F. Manetti, J. Esté, I. Clotet-Codina, G. Maga, R. Cancio, M. Botta and F. Corelli, Synthesis and biological investigation of S-aryl-S-DABO derivatives as HIV-1 inhibitors, Bioorg. Med. Lett. 16 (2006) 3541-3544; DOI: 10.1016/j.bmc.2006.03.080.

3. A. B. Adnan, T. Y. Hesham, A. F. Sherif and M. B. Azza, Design and synthesis of some substituted 1H-pyrazolyl-thiazolo[4,5-d]pyrimidines as anti-inflammatory-antimicrobial agents, Eur. J. Med. Chem. 38 (2003) 27-36; DOI: 10.1016/S0223-5234(02)00009-0.

4. E. A. Abdel-Galil, M. M. Ashraf, F. M. Salwa, A. A. Nagla and G. H. Abu El-Fotooh, Anticancer activities of some newly synthesized pyridine, pyrane and pyrimidine derivatives, Bioorg. Med. Chem. 14 (2006) 5481-5488; DOI: 10.1016/J.bmc.2006.04.045.

5. M. T. Cocco, C. Congiu, V. Lilliu and V. Onnis, Synthesis and in vitro antitumoral activity of new hydrazinopyrimidine-5-carbonitrile derivatives, Bioorg. Med. Chem. 14 (2006) 366-372; DOI: 10.1016/j.bmc.2005.08.012.

6. N. Zhang, S. Ayral-Kaloustian, T. Nguyen, R. Hernandez and C. Beyer, 2-Cyanoaminopyrimidines as a class of antitumor agents that promote tubulin polymerization, Bioorg. Med. Chem. Lett. 17 (2007) 3003-3005; DOI: 10.1016/j.bmcl. 2007.03.070.

7. N. Sirisoma, S. Kasibhatla, B. Nguyen, A. Pervin, Y. Wang, G. Claassen, B. Tseng, J. Drewe and S. Xiong, Discovery of substituted 4-anillino-2-(2-pyridyl)pyrimidines as a new series of apoptosis inducers using a cell- and caspase-based high throughput screening assay. Part I: Struc- 
ture-activity relathionships of the 4-anilino group, Bioorg. Med. Chem. 14 (2006) 7761-7773; DOI: 10.1016/j.bmc.2006.08.002.

8. P. Sharma, N. Rane and V. K. Gurram, Synthesis and QSAR studies of pyrimido[4,5-d]pyrimidine-2,5-dione derivatives as potential antimicrobial agents, Bioorg. Med. Chem. Lett. 14 (2004) 4185-4190; DOI: 10.1016/j.bmcl. 2004.06.014.

9. K. S. Jain, T. S. Chitre, P. B. Miniyar, M. K. Kathiravan, V. S. Bendre,V. S. Veer, S. R. Shahane and C. J. Shishoo, Biological and medicinal significance of pyrimidines, Curr. Sci. 90 (2006) 793-803.

10. O. A. Fathalla, S. M. Awad and M. S. Mohamed, Synthesis of new 2-thiouracil-5-sulphonamide derivatives with antibacterial and antifungal activity, Arch. Pharm. Res. 28 (2005) 1205-1212; DOI: $10.1007 /$ bf02978199.

11. O. A. Fathalla,W. A. Zaghary, H. H. Radwan, S. A. Awad, and M. S. Mohamed, Synthesis of new 2-thiouracil-5-sulphonamide derivatives with expected biological activity, Arch. Pharm. Res. 25 (2002) 258-269; DOI: 10.1007 bf 02976623.

12. Y. Ding, J. Girardet, K. L. Smith, G. L. Prigaro, J. Z. Wu and, N. Yao, Parallel synthesis of 5-cyano-6-aryl-2-thiouracil derivatives as inhibitors for hepatitis C viral NS5B RNA-dependent RNA polymerase, Bioorg. Chem. 34 (2006) 26-38; DOI: 10.1016/j.bioorg.2005.10.001.

13. N. Agarwal, P. Srivastava, S. K. Raghuwanshi, D. N. Upadhyay, S. Sinha, P. K. Shukla and V. J. Ram, Chloropyrimidines as a new class of antimicrobial agents, Bioorg. Med. Chem. 10 (2002) 869-874; DOI: 10.1016/S0968-0896(01)00374-1.

14. A. W. Bauer, M. M. Kirby, J. C. Sherris and M. Turck, Antibiotic susceptibility testing by a standardized single disc method, Am. J. Clin. Pathol. 45 (1996) 493-496.

15. A. A. Aly, Synthesis and antimicrobial activity of some annelated quinazoline derivatives, J. Chin. Chem. Soc. 54 (2007) 437-446.

\section{$S A \check{Z} E T A K$}

\section{Sinteza i antimikrobno vrednovanje nekoliko 6-aril-5-cijano-2-tiouracil derivata}

MOSAAD SAYED MOHAMED, SAMIR MOHAMED AWAD i NAGLAA MOHAMED AHMED

Polazeći iz etil-cijanoacetata, tiouree i odgovarajućeg aldehida sintetizirana je serija 6-aril-5-cijano-2-tiouracil derivata (1a-d), koji su potom upotrijebljeni za dobivanje tiouracil derivata (2a-d do 10a-d). Svim spojevima ispitano je antibakterijsko i antimikotsko djelovanje. Neki od sintetiziranih spojeva, 6-(4-fluorofenil)-4-okso-2-tiokso-1,2,3,4-tetrahidropirimidin-5-karboksamid (2a), 4-okso-2-tiokso-6-(3,4,5-trimetoksifenil)-1,2,3,4-tetrahidropirimidin-5-karboksamid (2d), 6-(4-fluorofenil)-4-hidrazino-2-tiokso-1,2-dihidropirimidin-5-kabonitril (7a) i 4-hidrazino-2-tiokso-6-(3,4,5-trimetoksifenil)-1,2-dihidropirimidin-5-karbonitril (7d) imaju značajno antimikrobno djelovanje.

Ključne riječi: 6-aril-5-cijano-2-tiouracil, antibakterijsko djelovanje, antimikotsko djelovanje

Organic Chemistry Department, Faculty of Pharmacy, Helwan University, Cairo, Egypt 